CRYSTALLOGRAPHIC COMMUNICATIONS

ISSN 2056-9890

Received 27 January 2020

Accepted 14 February 2020

Edited by C. Massera, Università di Parma, Italy

Keywords: crystal structure; pyrazine; tetrakissubstituted; $\mathrm{C}-\mathrm{H} \cdots \pi$ interactions; offset $\pi-\pi$ interactions; Hirshfeld surface analysis.

CCDC references: $1984024 ; 1984023$; 1984022

Supporting information: this article has supporting information at journals.iucr.org/e

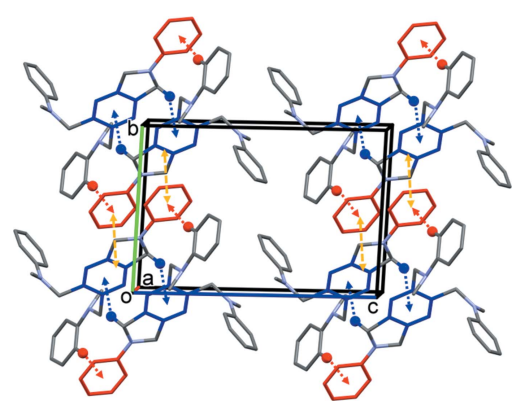

\section{Crystal structures and Hirshfeld surface analyses of two new tetrakis-substituted pyrazines and a degredation product}

\author{
Ana Tesouro Vallina ${ }^{a}$ and Helen Stoeckli-Evans ${ }^{\mathrm{b} *}$ \\ ${ }^{\mathbf{a}}$ Institute of Chemistry, University of Neuchâtel, Av. de Bellvaux 15, CH-2000 Neuchâtel, Switzerland, and ${ }^{\mathbf{b}}$ Institute of \\ Physics, University of Neuchâtel, rue Emile-Argand 11, CH-2000 Neuchâtel, Switzerland. *Correspondence e-mail: \\ helen.stoeckli-evans@unine.ch
}

The two new tetrakis-substituted pyrazines, $1,1^{\prime}, 1^{\prime \prime}, 1^{\prime \prime \prime}$-(pyrazine-2,3,5,6-tetrayl) tetrakis $\left(N, N\right.$-dimethylmethanamine), $\mathrm{C}_{16} \mathrm{H}_{32} \mathrm{~N}_{6},(\mathrm{I})$ and $N, N^{\prime}, N^{\prime \prime}, N^{\prime \prime \prime}$-[pyrazine2,3,5,6-tetrayltetrakis(methylene)] tetrakis( $N$-methylaniline), $\mathrm{C}_{36} \mathrm{H}_{40} \mathrm{~N}_{6}, \quad$ (II), both crystallize with half a molecule in the asymmetric unit; the whole molecules are generated by inversion symmetry. There are weak intramolecular $\mathrm{C}-\mathrm{H} \cdots \mathrm{N}$ hydrogen bonds present in both molecules and in (II) the pendant $\mathrm{N}$ methylaniline rings are linked by a $\mathrm{C}-\mathrm{H} \cdots \pi$ interaction. The degredation product, $\quad N, N^{\prime}$-[(6-phenyl-6,7-dihydro-5H-pyrrolo[3,4-b]pyrazine-2,3-diyl)bis(methylene)]bis( $N$-methylaniline), $\mathrm{C}_{28} \mathrm{H}_{29} \mathrm{~N}_{5}$, (III), was obtained several times by reacting (II) with different metal salts. Here, the 6-phenyl ring is almost coplanar with the planar pyrrolo[3,4- $b]$ pyrazine unit (r.m.s. deviation = $0.029 \AA$ ), with a dihedral angle of $4.41(10)^{\circ}$ between them. The two $N$-methylaniline rings are inclined to the planar pyrrolo $[3,4-b]$ pyrazine unit by $88.26(10)$ and $89.71(10)^{\circ}$, and to each other by $72.56(13)^{\circ}$. There are also weak intramolecular $\mathrm{C}-\mathrm{H} \cdots \mathrm{N}$ hydrogen bonds present involving the pyrazine ring and the two $N$-methylaniline groups. In the crystal of (I), there are no significant intermolecular contacts present, while in (II) molecules are linked by a pair of $\mathrm{C}-\mathrm{H} \cdots \pi$ interactions, forming chains along the $c$-axis direction. In the crystal of (III), molecules are linked by two pairs of $\mathrm{C}-\mathrm{H} \cdots \pi$ interactions, forming inversion dimers, which in turn are linked by offset $\pi-\pi$ interactions [intercentroid distance $=3.8492(19) \AA$ ] , forming ribbons along the $b$-axis direction.

\section{Chemical context}

Tetrakis-substituted pyrazines, which are potential bistridentate ligands, have been used in coordination chemistry since the 1980 's, to form not only mononuclear and binuclear complexes but also multi-dimensional coordination polymers. A search of the Cambridge Structural Database (CSD, Version 5.41, last update November 2019; Groom et al., 2016) reveals that the principal tetrakis-substituted pyrazine ligands that have been used are 2,3,5,6-tetrakis(pyridin-2-yl)pyrazine, which was first synthesized by Goodwin \& Lions (1959), and 2,3,5,6-pyrazinetetracarboxylic acid, which was first synthesized by Wolff at the end of the 19th century (Wolff, 1887, 1893). Since then the coordination chemistry of only a small number of tetrakis-substituted pyrazines has been studied, for example tetrakis(aminomethyl)pyrazine (Ferigo et al., 1994) and, more recently, the new ligand 2,3,5,6-tetrakis(4-carboxyphenyl) pyrazine, which has been shown to be extremely successful in forming metal-organic frameworks (Jiang et al., 2017; Wang et al., 2019). 
<smiles>CN(C)Cc1nc(CN(C)C)c(CN(C)C)nc1CN(C)C</smiles>

(I)<smiles>CN(Cc1nc2c(nc1CN(C)c1ccccc1)CN(c1ccccc1)C2)c1ccccc1</smiles>

(III)<smiles>CN(Cc1nc(CN(C)c2ccccc2)c(CN(C)c2ccccc2)nc1CN(C)c1ccccc1)c1ccccc1</smiles>

(II)

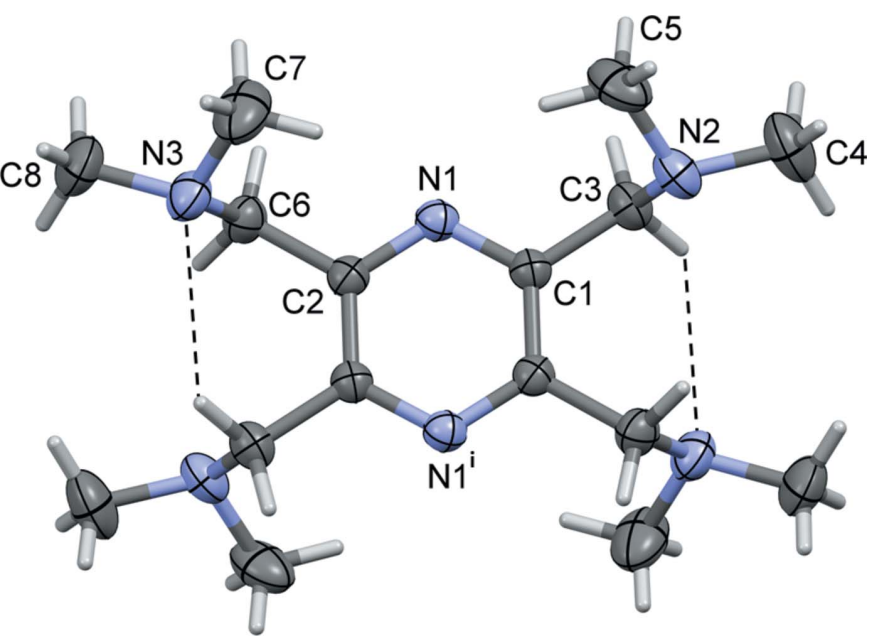

Figure 1

A view of the molecular structure of compound (I), with atom labelling [symmetry code: (i) $-x,-y,-z+1$ ]. Displacement ellipsoids are drawn at the $30 \%$ probability level. Intramolecular $\mathrm{C}-\mathrm{H} \cdots \mathrm{N}$ interactions (Table 1) are shown as dashed lines.

ving a methylaniline ring and the adjacent pyrazine $\mathrm{N}$ atom, and the methylaniline substituents in positions 2,6 (and 3,5)

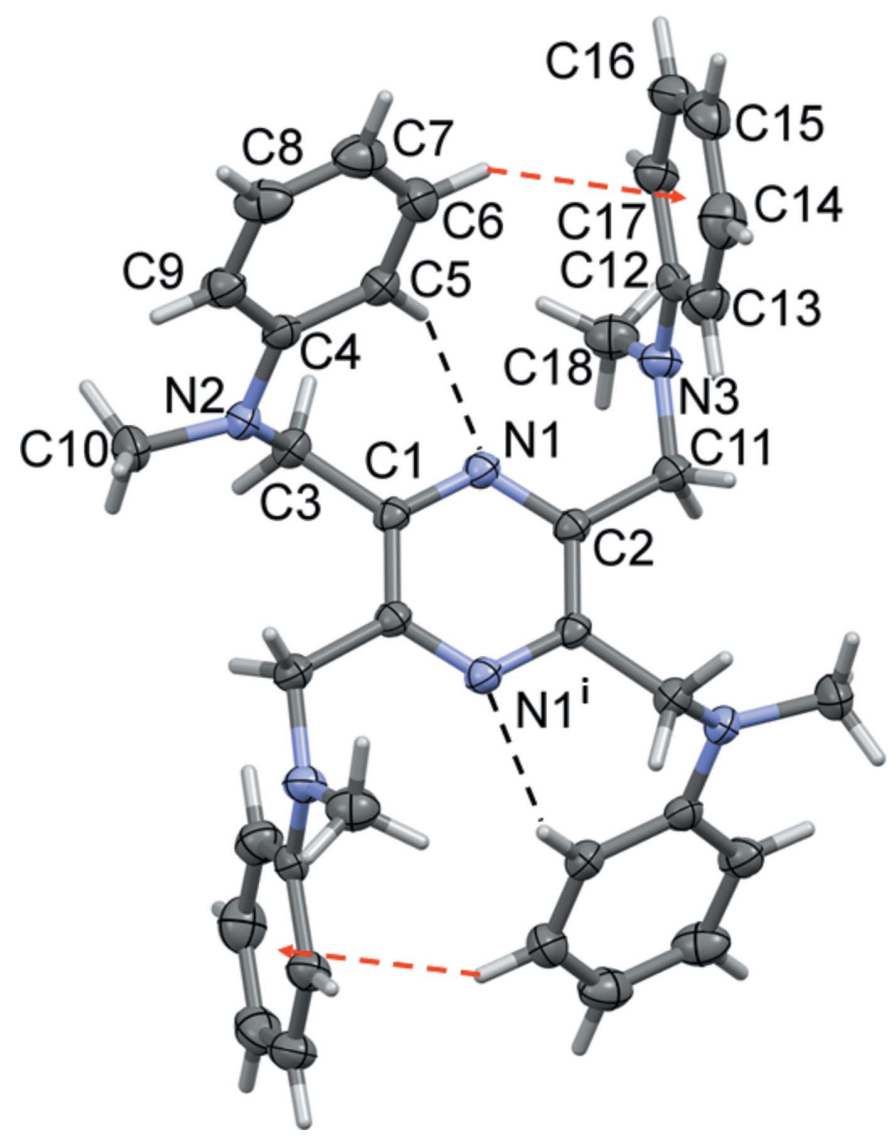

Figure 2

A view of the molecular structure of compound (II), with atom labelling [symmetry code: (i) $-x+1,-y+1,-z+2$ ]. Displacement ellipsoids are drawn at the $30 \%$ probability level. Intramolecular $\mathrm{C}-\mathrm{H} \cdots \mathrm{N}$ interactions (Table 2) are shown as dashed lines and the intramolecular $\mathrm{C}-$ $\mathrm{H} \cdots \pi$ interactions (Table 2) as red dashed arrows. 
Table 1

Hydrogen-bond geometry $\left(\AA{ }^{\circ}\right)$ for (I).

\begin{tabular}{lllll}
\hline$D-\mathrm{H} \cdots A$ & $D-\mathrm{H}$ & $\mathrm{H} \cdots A$ & $D \cdots A$ & $D-\mathrm{H} \cdots A$ \\
\hline $\mathrm{C} 3-\mathrm{H} 3 A \cdots \mathrm{N} 3^{\mathrm{i}}$ & 0.97 & 2.62 & $3.261(4)$ & 124 \\
\hline
\end{tabular}

Symmetry code: (i) $-x,-y,-z+1$.

Table 2

Hydrogen-bond geometry $\left(\AA{ }^{\circ}{ }^{\circ}\right)$ for (II).

$C g 2$ and $C g 3$ are the centroids of rings $\mathrm{C} 4-\mathrm{C} 9$ and $\mathrm{C} 12-\mathrm{C} 17$, respectively.

\begin{tabular}{lllll}
\hline$D-\mathrm{H} \cdots A$ & $D-\mathrm{H}$ & $\mathrm{H} \cdots A$ & $D \cdots A$ & $D-\mathrm{H} \cdots A$ \\
\hline $\mathrm{C} 5-\mathrm{H} 5 \cdots \mathrm{N} 1$ & 0.93 & 2.50 & $3.331(3)$ & 149 \\
$\mathrm{C} 6-\mathrm{H} 6 \cdots C g 3$ & 0.93 & 2.99 & $3.804(3)$ & 147 \\
$\mathrm{C} 3-\mathrm{H} 3 A \cdots \mathrm{Cg} 2^{\mathrm{i}}$ & 0.97 & 2.83 & $3.561(2)$ & 133 \\
\hline
\end{tabular}

Symmetry code: (i) $-x+1,-y+1,-z+1$.

Table 3

Hydrogen-bond geometry $\left(\AA,^{\circ}\right)$ for (III).

$\mathrm{Cg} 2$ and $\mathrm{Cg} 3$ are the centroids of rings $\mathrm{N} 1 / \mathrm{N} 2 / \mathrm{C} 1-\mathrm{C} 4$ and $\mathrm{C} 7-\mathrm{C} 12$, respectively.

\begin{tabular}{lllll}
\hline$D-\mathrm{H} \cdots A$ & $D-\mathrm{H}$ & $\mathrm{H} \cdots A$ & $D \cdots A$ & $D-\mathrm{H} \cdots A$ \\
\hline $\mathrm{C} 15-\mathrm{H} 15 \cdots \mathrm{N} 4$ & 0.93 & 2.61 & $3.542(3)$ & 175 \\
$\mathrm{C} 28-\mathrm{H} 28 B \cdots \mathrm{N} 2$ & 0.96 & 2.59 & $3.323(3)$ & 133 \\
$\mathrm{C} 6-\mathrm{H} 6 B \cdots C g 2^{\mathrm{i}}$ & 0.97 & 2.82 & $3.601(2)$ & 138 \\
$\mathrm{C} 23-\mathrm{H} 23 \cdots C g 3^{\mathrm{i}}$ & 0.93 & 2.97 & $3.881(3)$ & 168 \\
\hline
\end{tabular}

Symmetry code: (i) $-x+1,-y+2,-z$.

are linked by an intramolecular C6 $-\mathrm{H} 6 \cdots \pi$ interaction (Fig. 2 and Table 2).

The molecular structure of compound (III) is illustrated in Fig. 3. One side of the molecule has been transformed into a pyrrolo unit fused to the pyrazine ring. The 6-phenyl ring (C7$\mathrm{C} 12)$ is almost coplanar with the planar pyrrolo[3,4- $b]$ pyrazine unit (N1-N3/C1-C6; r.m.s. deviation = $0.029 \AA$ ), forming a dihedral angle of $4.41(10)^{\circ}$. On the other side of the molecule, the two adjacent $N$-methylaniline rings (C14-C19 and C22$\mathrm{C} 27)$ are inclined to the planar pyrrolo[3,4- $b]$ pyrazine unit by

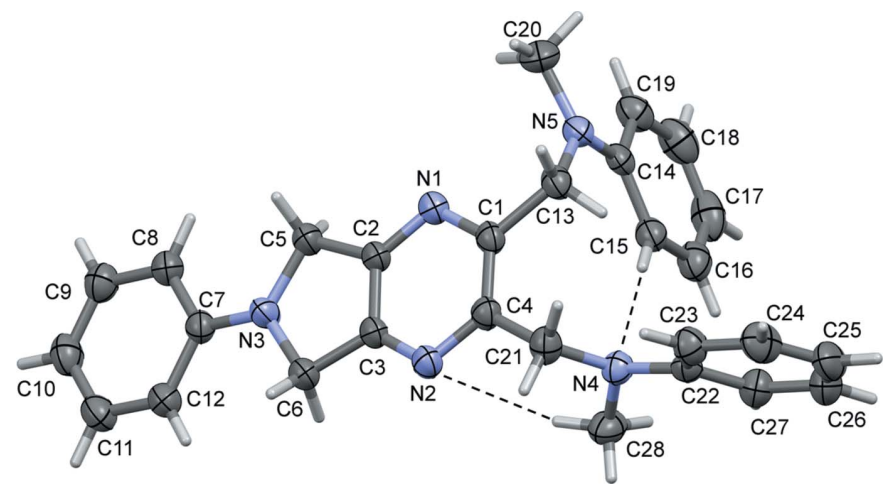

Figure 3

A view of the molecular structure of compound (III), with atom labelling. Displacement ellipsoids are drawn at the $30 \%$ probability level. Intramolecular $\mathrm{C}-\mathrm{H} \cdots \mathrm{N}$ interactions (Table 3) are shown as dashed lines.

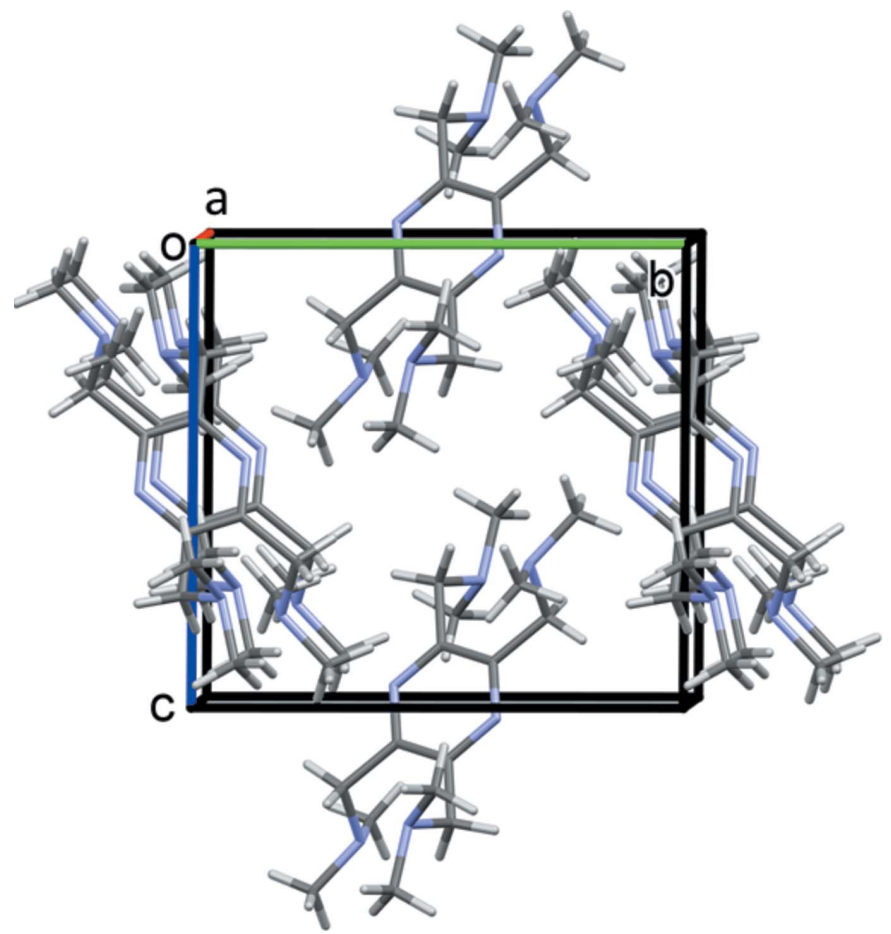

Figure 4

A view along the $a$ axis of the crystal packing of compound (I).

$88.26(10)$ and $89.71(10)^{\circ}$, and to each other by $72.56(13)^{\circ}$. There are also weak intramolecular $\mathrm{C}-\mathrm{H} \cdots \mathrm{N}$ hydrogen bonds present involving the pyrazine ring and the two $\mathrm{N}$ methylaniline groups (Fig. 3 and Table 3).

\section{Supramolecular features}

In the crystal of (I), there are no significant intermolecular interactions present (Fig. 4).

In the crystal of (II), molecules are linked by a pair of $\mathrm{C}-$ $\mathrm{H} \cdots \pi$ interactions, forming chains that propagate along the [001] direction (Fig. 5 and Table 2).

In the crystal of (III), molecules are linked by two pairs of $\mathrm{C}-\mathrm{H} \cdots \pi$ interactions, forming inversion dimers. Offset $\pi-\pi$ interactions link the dimers to form ribbons propagating along the [010] direction; see Fig. 6 and Table 3. The offset $\pi-\pi$ interaction, $\mathrm{Cg} 3 \cdots \mathrm{Cg} 6{ }^{\mathrm{ii}}$, where $\mathrm{Cg} 3$ and $\mathrm{C} g 6$ are, respectively, the centroids of the phenyl ring $(\mathrm{C} 7-\mathrm{C} 12)$ and the pyrrolo$[3,4-b]$ pyrazine ring system, has a centroid-centroid distance of $3.8492(14) \AA, \alpha=4.41(10)^{\circ}$, interplanar distances of

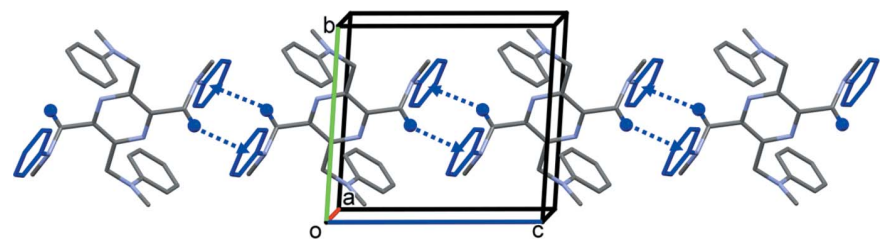

Figure 5

A view along the $a$ axis of the crystal packing of compound (II). The C3$\mathrm{H} 3 A \cdots \pi$ interactions (Table 2) are shown as blue dashed arrows, and for clarity, only $\mathrm{H}$ atom $\mathrm{H} 3 A$ (blue) has been included. 


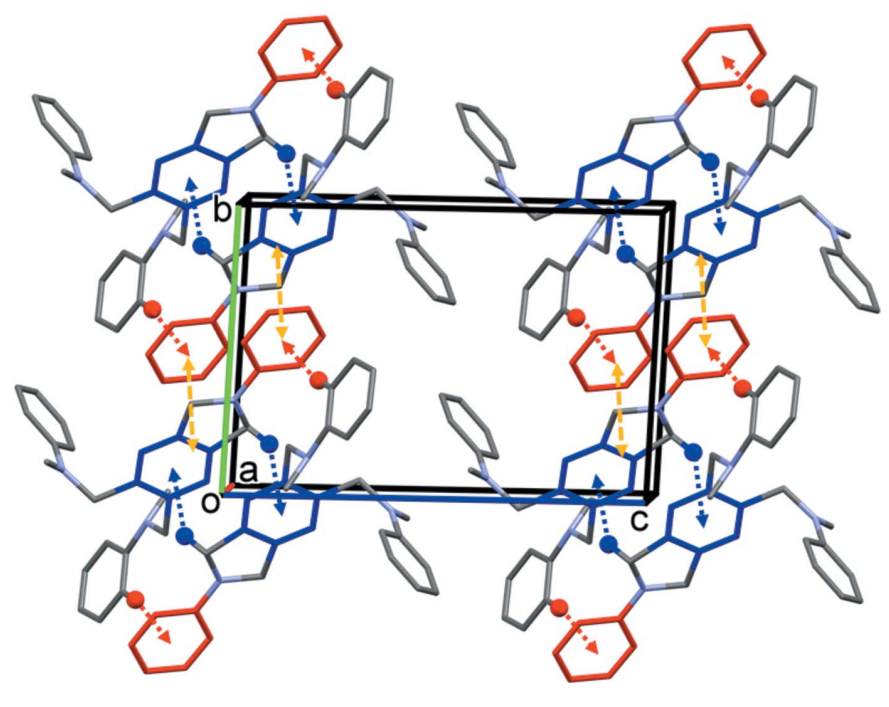

Figure 6

A view along the $a$ axis of the crystal packing of compound (III). The C$\mathrm{H} \cdots \pi$ interactions (Table 3 ) are shown as blue and red dashed arrows. For clarity, only the $\mathrm{H}$ atoms $\mathrm{H} 6 B$ (blue) and $\mathrm{H} 23$ (red) have been included. The offset $\pi-\pi$-interactions are shown as orange dashed double arrows.

3.6495 (14) and 3.5490 (7) $\AA$, with an offset of $1.49 \AA$ [symmetry code: (ii) $-x+1,-y+1,-z$ ].

\section{Hirshfeld surface analysis and two-dimensional fingerprint plots}

The Hirshfeld surface analysis (Spackman \& Jayatilaka, 2009) and the associated two-dimensional fingerprint plots (McKinnon et al., 2007) were performed with CrystalExplorer17 (Turner et al., 2017).

The Hirshfeld surfaces are colour-mapped with the normalized contact distance, $d_{\text {norm }}$, from red (distances shorter

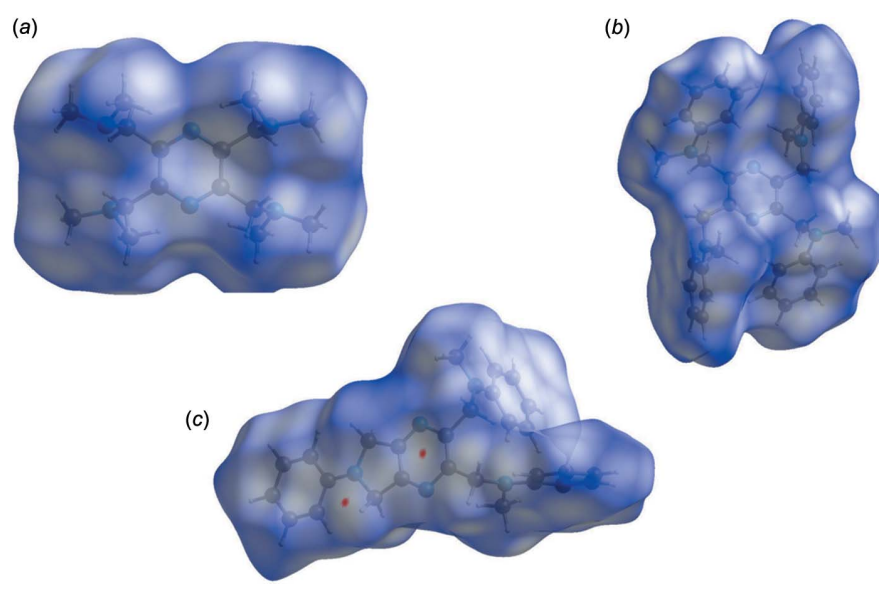

Figure 7

(a) The Hirshfeld surface of compound (I), mapped over $d_{\text {norm }}$ in the colour range -0.7519 to 1.6997 a.u., $(b)$ the Hirshfeld surface of compound (II), mapped over $d_{\text {norm }}$ in the colour range -0.7519 to 1.6997 a.u. and (c) the Hirshfeld surface of compound (III), mapped over $d_{\text {norm }}$ in the colour range -0.7519 to 1.6997 a.u.. (a)
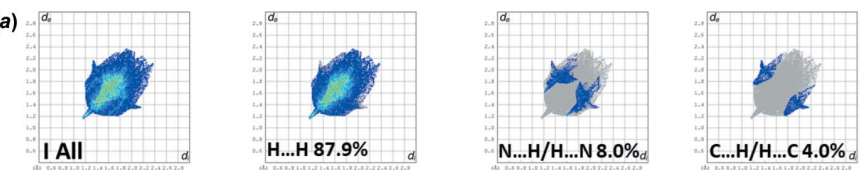

(b)

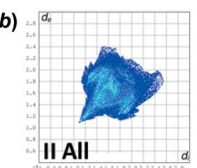

(c)

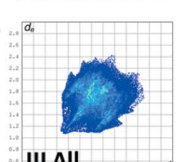

III All
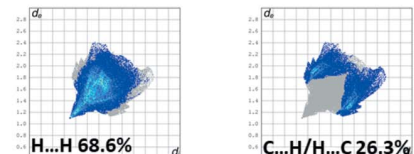

C...H/H...C $26.3 \%$
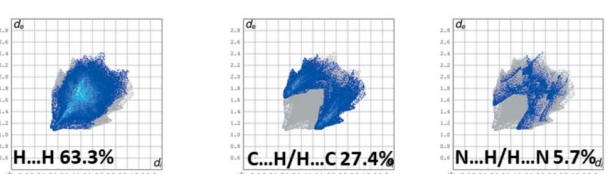

Figure 8

(a) The full two-dimensional fingerprint plot for compound (I), and fingerprint plots delineated into $\mathrm{H} \cdots \mathrm{H}, \mathrm{N} \cdots \mathrm{H} / \mathrm{H} \cdots \mathrm{N}$ and $\mathrm{C} \cdots \mathrm{H} / \mathrm{H} \cdots \mathrm{C}$ contacts, $(b)$ the full two-dimensional fingerprint plot for compound (II), and fingerprint plots delineated into $\mathrm{H} \cdots \mathrm{H}, \mathrm{C} \cdots \mathrm{H} / \mathrm{H} \cdots \mathrm{C}$ and $\mathrm{N} \cdots \mathrm{H} /$ $\mathrm{H} \cdots \mathrm{N}$ contacts and $(c)$ the full two-dimensional fingerprint plot for compound (III), and fingerprint plots delineated into $\mathrm{H} \cdots \mathrm{H}, \mathrm{C} \cdots \mathrm{H} /$ $\mathrm{H} \cdots \mathrm{C}$ and $\mathrm{N} \cdots \mathrm{H} / \mathrm{H} \cdots \mathrm{N}$ contacts.

than the sum of the van der Waals radii) through white to blue (distances longer than the sum of the van der Waals radii). The Hirshfeld surfaces (HS) of the title compounds, mapped over $d_{\text {norm }}$, are given in Fig. 7. It is evident from Figs. $7 a$ and $7 b$ that there are no contact distances shorter than the sum of the van der Waals radii in the crystals of either compounds (I) or (II). For compound (III) (Fig. 7c), two small red spots indicate the presence of weak C $\cdots \mathrm{H}$ contacts (see Table 3 ).

The two-dimensional fingerprint plots for the title compounds are given in Fig. 8. They reveal, as expected, that the principal contributions to the overall surface involve $\mathrm{H} \cdots \mathrm{H}$ contacts at $87.9 \%$ for (I) (Fig. $8 a$ ), $68.6 \%$ for (II) (Fig. $8 b$ ), and $63.3 \%$ for (III) (Fig. $8 c$ ). The second most important contribution to the HS for compound (I) is from the $\mathrm{N} \cdots \mathrm{H} / \mathrm{H} \cdots \mathrm{N}$ contacts at $8.0 \%$; for compounds (II) and (III) the second most significant contributions are from the $\mathrm{C} \cdots \mathrm{H} /$ $\mathrm{H}$. . C contacts at 26.3 and $27.4 \%$, respectively. For compound (I), the third most important contribution to the HS is from the $\mathrm{C} \cdots \mathrm{H} / \mathrm{H} \cdots \mathrm{C}$ contacts at $4.0 \%$, while for compounds (II) and (III) it is from the $\mathrm{N} \cdots \mathrm{H} / \mathrm{H} \cdots \mathrm{N}$ contacts at 2.6 and $5.7 \%$, respectively. All other atom $\cdots$ atom contacts contribute less that $2 \%$ to the HS for all three compounds.

\section{Database survey}

A search of the CSD (Version 5.41, last update November 2019; Groom et al., 2016) for the structure of 2,3,5,6-tetrakis(pyridin-2-yl)pyrazine gave 289 hits, of which 91 structures are polymeric. The first polymeric compound to be reported in 1995 was for a trinuclear cobalt(II) one-dimensional coordination polymer, catena-[bis $\left(\mu_{2}\right.$-chloro)acetonitriletetrachloro[2,3,5,6-tetrakis(2-pyridyl)pyrazine]tricobalt(II)] (CSD refcode TUPWAC; Constable et al., 1995).

A search for the structure of 2,3,5,6-pyrazinetetracarboxylic acid gave 92 hits, of which 64 are polymeric. Here, the first 
polymeric compound to be reported in 1986 was for a binuclear iron(II) polymer chain, catena- $\left[\mu_{2}-(2,5\right.$-dicarboxypyrazine-3,6-dicarboxylato- $N, O$ ) trans-diaquadiiron(II)] dihydrate (DUWROC; Marioni et al., 1986).

A search for the structure of tetrakis(aminomethyl)pyrazine yielded only eight hits, of which five compounds are polymeric; see for example catena-[ $\mu_{2}$-[tetrakis(aminomethyl)pyrazine- $\left.N, N^{\prime}, N^{\prime \prime}\right]$ manganese dichloride dihydrate] (PITXEV; Ferigo et al., 1994), and catena-[[ $\mu_{2}-2,3,5,6$-tetrakis(aminomethyl)pyrazine $]$ bis $\left(\mu_{2}\right.$-chloro)dichlorodicopper hydrate] (PITXIZ; Ferigo et al., 1994).

Recently a new ligand, 2,3,5,6-tetrakis(4-carboxyphenyl pyrazine), has been shown to be extremely successful in forming 17 metal-organic frameworks (MOFs). It was designed by Jiang and coworkers (Jiang et al., 2017) who produced the first MOF using this ligand, viz. catena-[( $\mu$ $4,4^{\prime}, 4^{\prime \prime}, 4^{\prime \prime \prime}$-pyrazine-2,3,5,6-tetrabenzoato)bis( $N, N$-dimethylformamide)dizinc unknown solvate] (NAWXER; Jiang et al., 2017). Since then the ligand has been used by a number of groups, and the most recent MOF to be published is catena-[( $\mu$-4,4'-bipyridine $)$ bis $(\mu$-hydroxo $)$ bis $[\mu$-dihydrogen $4,4^{\prime}, 4^{\prime \prime}, 4^{\prime \prime \prime}$-(pyrazine-2,3,5,6-tetrayl)tetrabenzoato]trinickel unknown solvate] (HOQTUF; Wang et al., 2019).

In relation to the structure of compound (III), a search for the substructure pyrrolo[3,4-b]pyrazine yielded only two hits. They concern dipyrrolo[ $\left[3,4-b: 3^{\prime}, 4^{\prime}-e\right]$ pyrazine structures that possess inversion symmetry, viz. 2,6-dibenzyl-1,2,3,5,6,7hexahydrodipyrrolo[3,4-b:3',4'-e]pyrazine (EXUHIO; Gasser \& Stoeckli-Evans, 2004) and 2,6-bis(4-methoxybenzyl)1,2,3,5,6,7-hexahydrodipyrrolo[3,4-b:3', $\left.4^{\prime}-e\right]$ pyrazine (EXUHOU; Gasser \& Stoeckli-Evans, 2004). They were prepared during attempts to form 1,2,3,5,6,7-hexahydro-2,4,6,8-tetraaza-s-indacene by reacting 2,3,5,6-tetrakis(bromomethyl)pyrazine (Ferigo et al., 1994; TOJXUN: Assoumatine \& Stoeckli-Evans, 2014) with the corresponding amines. In contrast to (III), where the pyrrolo ring is planar (r.m.s. deviation $=0.029 \AA$ ) and inclined by only $2.00(12)^{\circ}$ to the pyrazine ring, here the pyrrolo groups have envelope conformations with the pyrrolo $\mathrm{N}$ atoms as the flaps. Their mean planes are inclined to the pyrazine ring by $7.88(16)^{\circ}$ in EXUHIO and by 8.05 (7) ${ }^{\circ}$ in EXUHOU.

\section{Synthesis and crystallization}

Synthesis of $1,1^{\prime}, \mathbf{1}^{\prime \prime}, \mathbf{1}^{\prime \prime \prime}$-(pyrazine-2,3,5,6-tetrayl) tetrakis $(N, N$ dimethylmethanamine) (I):

A large excess of dimethyl amine hydrochloride in water was neutralized with $\mathrm{NaOH}$ in an ice bath. $\mathrm{Me}_{2} \mathrm{NH}$ formed in situ as a gas and was directly condensed in a round-bottom flask in an acetone/liquid $\mathrm{N}_{2}$ bath at about $213 \mathrm{~K}$ using a weak vacuum. Once a sufficient quantity of liquid amine had formed, a solution of 2,3,5,6-tetrakis(bromomethyl)pyrazine $(0.4530 \mathrm{~g}, 1 \mathrm{mmol})$ in $50 \mathrm{ml}$ of $\mathrm{CH}_{2} \mathrm{Cl}_{2}$ was added dropwise at low temperature ( $c a 243 \mathrm{~K}$ ). The reaction was left for about $4 \mathrm{~h}$, allowing the temperature rise to RT. The excess amine was allowed to evaporate off before the solvent was gassed off. The residue obtained was dissolved in $40 \mathrm{ml}$ of $\mathrm{MeOH}$ and passed through a resin column (15 g of Dowex $1 \mathrm{X} 8$ ) previously charged with $\mathrm{OH}^{-}$ions in order to exchange the $\mathrm{HBr}$ molecules, still attached to the ligand, by $\mathrm{H}_{2} \mathrm{O}$ molecules. About $150 \mathrm{ml}$ were used as eluent. Solvent evaporation yielded $0.27 \mathrm{~g}(87 \%)$ of a light-yellow powder of compound (I). Colourless block-like crystals were obtained by slow diffusion of hexane into a solution of the ligand in dichloromethane.

${ }^{1} \mathrm{H}$ NMR $\left(\mathrm{CDCl}_{3}, 200 \mathrm{MHz}, \mathrm{ppm}\right): 3.65\left(s, 8 \mathrm{H}, \mathrm{CH}_{2}\right), 2.15(s$, $\left.12 \mathrm{H}, \mathrm{CH}_{3}\right) .{ }^{13} \mathrm{C}$ NMR $\left(\mathrm{D}_{2} \mathrm{O}, 400 \mathrm{MHz}, \mathrm{ppm}\right): 152.16,62.53$, 46.54. IR (KBr pellet, $\left.\mathrm{cm}^{-1}\right): 2974(s), 2942(s), 2854(\mathrm{~m}), 2820$ $(v s), 2772(v s), 1635(b), 1456(s), 1414(m), 1348(s), 1259(s)$, $1204(\mathrm{~m}), 1168(\mathrm{~m}), 1027(\mathrm{vs}), 987(\mathrm{~m}), 841(\mathrm{~s})$. MS (EI, $70 \mathrm{eV})$, $\mathrm{m} / \mathrm{z}: 310\left(\mathrm{MH}^{+}\right), 264,178$. Anal. for $\mathrm{C}_{16} \mathrm{H}_{32} \mathrm{~N}_{6}\left(308.5 \mathrm{~g} \mathrm{~mol}^{-1}\right)$ Calculated (\%) C 62.30, H 10.46, N 27.24. Found (\%) C 61.86, H 10.73, N 27.50.

Synthesis of $N, N^{\prime}, N^{\prime \prime}, N^{\prime \prime \prime}$-[pyrazine-2,3,5,6-tetrayltetrakis(methylene)]tetrakis( $N$-methylaniline) (II):

A solution of 2,3,5,6-tetrakis(bromomethyl)pyrazine $(0.4530 \mathrm{~g}, 1 \mathrm{mmol})$ in $35 \mathrm{ml}$ of $\mathrm{CH}_{3} \mathrm{CN}$ was added dropwise to a suspension of $N$-methylaniline $(1.2 \mathrm{ml}, 10 \mathrm{mmol})$ and $\mathrm{Na}_{2} \mathrm{CO}_{3}(5.3 \mathrm{~g}, 50 \mathrm{mmol})$ in $25 \mathrm{ml}$ of $\mathrm{CH}_{3} \mathrm{CN}$. The colour changed immediately from light to deep yellow. The mixture was refluxed for $c a 2 \mathrm{~h}$, followed by TLC and then cooled to RT. The white precipitate $\left(\mathrm{NaBr}\right.$ and excess $\left.\mathrm{Na}_{2} \mathrm{CO}_{3}\right)$ was filtered off and the filtrate was evaporated under vacuum. The residue was dissolved in hexane and the insoluble yellow powder obtained was recovered, washed with more hexane and then dried to yield $0.335 \mathrm{~g}(60 \%)$ of compound (II). Palegreenish-yellow block-like crystals were obtained by slow evaporation of a $\mathrm{CDCl}_{3}$ solution of (II) in an NMR tube.

${ }^{1} \mathrm{H}$ NMR $\left(\mathrm{CDCl}_{3}, 200 \mathrm{MHz}, \mathrm{ppm}\right): 7.14(t, 8 \mathrm{H}, \mathrm{ph}), 6.68(\mathrm{~m}$, $12 \mathrm{H}, \mathrm{ph}), 4.58\left(s, 8 \mathrm{H}, \mathrm{CH}_{2}\right), 2.79\left(s, 12 \mathrm{H}, \mathrm{CH}_{3}\right) .{ }^{13} \mathrm{C} \mathrm{NMR}$ $\left(\mathrm{CD}_{3} \mathrm{OD}, 400 \mathrm{MHz}, \mathrm{ppm}\right): 149.64,149.31,128.94,116.92$, 113.17, 54.75, 39.46. IR (KBr pellet, $\left.\mathrm{cm}^{-1}\right): 2926(w), 1601(s)$, $1508(v s), 1446(m), 1377(m), 1366(m), 1313(m), 1257(m)$, $1212(m), 1117(w), 993(w), 820(w), 745(s), 689(m)$. MS (EI, $70 \mathrm{eV}), m / z: 594\left(M \mathrm{~K}^{+}\right), 374,291$. Analysis for $\mathrm{C}_{36} \mathrm{H}_{40} \mathrm{~N}_{6}$ $\left(556.7 \mathrm{~g} \mathrm{~mol}^{-1}\right.$ ) Calculated (\%) C 77.66, H 7.24, N 15.09. Found (\%) C 76.82, H 7.19, N 15.07.

Synthesis of $N, N^{\prime}$-[(6-phenyl-6,7-dihydro-5H-pyrrolo[3,4-b]pyrazine-2,3-diyl)bis(methylene)]bis( $N$-methylaniline) (III):

Hexagonal pale-yellow plate-like crystals of compound (III) were obtained several times when reacting (II) with different metal salts, such as $\mathrm{Zn}\left(\mathrm{ClO}_{4}\right)_{2}$ (in excess), $\mathrm{MnCl}_{2} \cdot 4 \mathrm{H}_{2} \mathrm{O}$ and $\mathrm{Ni}(\mathrm{AcO})_{2} \cdot 4 \mathrm{H}_{2} \mathrm{O}$. No spectroscopic or other analytical data are available for this compound.

\section{Refinement}

Crystal data, data collection and structure refinement details are summarized in Table 4 . The $\mathrm{C}$-bound $\mathrm{H}$ atoms were included in calculated positions and treated as riding on their parent $\mathrm{C}$ atom: $\mathrm{C}-\mathrm{H}=0.93-0.97 \AA$ with $U_{\text {iso }}(\mathrm{H})=1.5 U_{\text {eq }}(\mathrm{C}-$ methyl) and $1.2 U_{\mathrm{eq}}(\mathrm{C})$ for other $\mathrm{H}$ atoms. Note for compound (III): using the Stoe IPDS I, a one-circle diffractometer, to 
Table 4

Experimental details.

(I) (II)

Crystal data

Chemical formula

$M_{\mathrm{r}}$

Crystal system, space group

Temperature (K)

$a, b, c(\AA)$

$\alpha, \beta, \gamma\left(^{\circ}\right)$

$V\left(\AA^{3}\right)$

$Z$

Radiation type

$\mu\left(\mathrm{mm}^{-1}\right)$

Crystal size (mm)

Data collection

Diffractometer

No. of measured, independent and

observed $[I>2 \sigma(I)]$ reflections

$R_{\text {int }}$

$(\sin \theta / \lambda)_{\max }\left(\AA^{-1}\right)$

$\mathrm{C}_{16} \mathrm{H}_{32} \mathrm{~N}_{6}$

308.47

Monoclinic, $P 2_{1} / n$

293

9.7577 (14), 10.348 (2), 9.9118 (16)

90, 101.663 (15), 90

$980.2(3)$

2

Mo $K \alpha$

0.07

$0.53 \times 0.53 \times 0.26$

Stoe-Siemens AED2, 4-circle

3347, 1818, 1111

0.055

0.605

$0.060,0.154,1.10$

1818

105

$\mathrm{H}$-atom parameters constrained $0.15,-0.12$

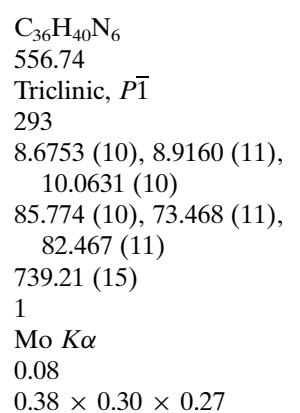

$0.38 \times 0.30 \times 0.27$

Stoe-Siemens AED2, 4-circle 5354, 2741, 1913

0.031

0.605

$0.049,0.108,1.12$

2741

193

$\mathrm{H}$-atom parameters constrained $0.13,-0.15$
(III)

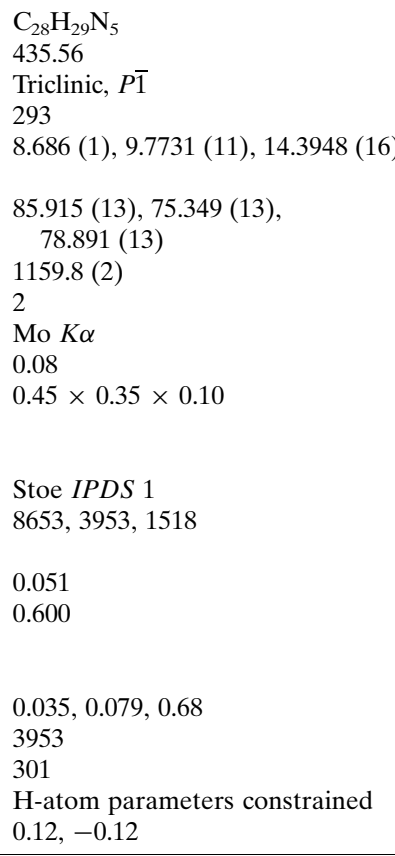

$\Delta \rho_{\max }, \Delta \rho_{\min }\left(\mathrm{e} \AA^{-3}\right)$

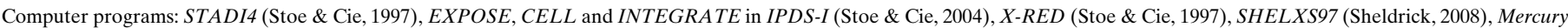
(Macrae et al., 2020), SHELXL2018/3 (Sheldrick, 2015), PLATON (Spek, 2020) and publCIF (Westrip, 2010).

measure data for the triclinic system often only $93 \%$ of the Ewald sphere is accessible. Hence, the diffrn_reflns_Laue_ measured_fraction_full of 0.939 is below the required minimum of 0.95 .

\section{Acknowledgements}

HSE is grateful to the University of Neuchâtel for their support over the years.

\section{Funding information}

Funding for this research was provided by: Swiss National Science Foundation, University of Neuchâtel.

\section{References}

Assoumatine, T. \& Stoeckli-Evans, H. (2014). Acta Cryst. E70, 51-53. Constable, E. C., Edwards, A. J., Phillips, D. \& Raithby, P. (1995). Supramol. Chem. 5, 93-95.

Ferigo, M., Bonhôte, P., Marty, W. \& Stoeckli-Evans, H. (1994). J. Chem. Soc. Dalton Trans. pp. 1549-1554.

Gasser, G. \& Stoeckli-Evans, H. (2004). Acta Cryst. C60, o514-0516. Goodwin, H. A. \& Lions, F. (1959). J. Am. Chem. Soc. 81, 6415-6422. Groom, C. R., Bruno, I. J., Lightfoot, M. P. \& Ward, S. C. (2016). Acta Cryst. B72, 171-179.
Jiang, Y., Sun, L., Du, J., Liu, Y., Shi, H., Liang, Z. \& Li, J. (2017). Cryst. Growth Des. 17, 2090-2096.

Macrae, C. F., Sovago, I., Cottrell, S. J., Galek, P. T. A., McCabe, P., Pidcock, E., Platings, M., Shields, G. P., Stevens, J. S., Towler, M. \& Wood, P. A. (2020). J. Appl. Cryst. 53, 226-235.

Marioni, P.-A., Stoeckli-Evans, H., Marty, W., Güdel, H.-U. \& Williams, A. F. (1986). Helv. Chim. Acta, 69, 1004-1011.

McKinnon, J. J., Jayatilaka, D. \& Spackman, M. A. (2007). Chem. Commun. pp. 3814-3816.

Sheldrick, G. M. (2008). Acta Cryst. A64, 112-122.

Sheldrick, G. M. (2015). Acta Cryst. C71, 3-8.

Spackman, M. A. \& Jayatilaka, D. (2009). CrystEngComm, 11, 19-32. Spek, A. L. (2020). Acta Cryst. E76, 1-11.

Stoe \& Cie (1997). STADI4 and X-RED. Stoe \& Cie GmbH, Darmstadt, Germany.

Stoe \& Cie (2004). IPDSI Bedienungshandbuch. Stoe \& Cie GmbH, Darmstadt, Germany.

Tesouro Vallina, A. (2001). PhD Thesis. University of Neuchâtel, Switzerland.

Turner, M. J., McKinnon, J. J., Wolff, S. K., Grimwood, D. J., Spackman, P. R., Jayatilaka, D. \& Spackman, M. A. (2017). CrystalExplorer17. University of Western Australia. http://hirshfeldsurface.net

Wang, L., Zou, R., Guo, W., Gao, S., Meng, W., Yang, J., Chen, X. \& Zou, R. (2019). Inorg. Chem. Commun. 104, 78-82.

Westrip, S. P. (2010). J. Appl. Cryst. 43, 920-925.

Wolff, L. (1887). Ber. Dtsch. Chem. Ges. 20, 425-433.

Wolff, L. (1893). Ber. Dtsch. Chem. Ges. 26, 721-725. 


\section{supporting information}

Acta Cryst. (2020). E76, 404-409 [https://doi.org/10.1107/S2056989020002133]

\section{Crystal structures and Hirshfeld surface analyses of two new tetrakis-substituted pyrazines and a degredation product}

\section{Ana Tesouro Vallina and Helen Stoeckli-Evans}

Computing details

Data collection: STADI4 (Stoe \& Cie, 1997) for (I), (II); EXPOSE in IPDS-I (Stoe \& Cie, 2004) for (III). Cell refinement: STADI4 (Stoe \& Cie, 1997) for (I), (II); CELL in IPDS-I (Stoe \& Cie, 2004) for (III). Data reduction: X-RED (Stoe \& Cie, 1997) for (I), (II); INTEGRATE in IPDS-I (Stoe \& Cie, 2004) for (III). For all structures, program(s) used to solve structure: SHELXS97 (Sheldrick, 2008); program(s) used to refine structure: SHELXL2018/3 (Sheldrick, 2015); molecular graphics: Mercury (Macrae et al., 2020); software used to prepare material for publication: SHELXL2018/3 (Sheldrick, 2015), PLATON (Spek, 2020) and publCIF (Westrip, 2010).

$1,1^{\prime}, 1^{\prime \prime}, 1^{\prime \prime \prime}$-(Pyrazine-2,3,5,6-tetrayl)tetrakis( $N, N$-dimethylmethanamine) (I)

Crystal data

$\mathrm{C}_{16} \mathrm{H}_{32} \mathrm{~N}_{6}$

$M_{r}=308.47$

Monoclinic, $P 2_{1} / n$

$a=9.7577(14) \AA$

$b=10.348(2) \AA$

$c=9.9118(16) \AA$

$\beta=101.663(15)^{\circ}$

$V=980.2(3) \AA^{3}$

$Z=2$

\section{Data collection}

Stoe-Siemens AED2, 4-circle diffractometer

Radiation source: fine-focus sealed tube Plane graphite monochromator $\omega / \wedge 2 \mathrm{q}$ scans

3347 measured reflections 1818 independent reflections

1111 reflections with $I>2 \sigma(I)$

Refinement

Refinement on $F^{2}$

Least-squares matrix: full

$R\left[F^{2}>2 \sigma\left(F^{2}\right)\right]=0.060$

$w R\left(F^{2}\right)=0.154$

$S=1.10$

1818 reflections

105 parameters
$F(000)=340$

$D_{\mathrm{x}}=1.045 \mathrm{Mg} \mathrm{m}^{-3}$

Mo $K \alpha$ radiation, $\lambda=0.71073 \AA$

Cell parameters from 22 reflections

$\theta=12.6-18.1^{\circ}$

$\mu=0.07 \mathrm{~mm}^{-1}$

$T=293 \mathrm{~K}$

Block, colourless

$0.53 \times 0.53 \times 0.26 \mathrm{~mm}$

$R_{\text {int }}=0.055$

$\theta_{\max }=25.5^{\circ}, \theta_{\min }=2.7^{\circ}$

$h=-11 \rightarrow 11$

$k=0 \rightarrow 12$

$l=-11 \rightarrow 11$

2 standard reflections every $60 \mathrm{~min}$ intensity decay: $1 \%$

0 restraints

Primary atom site location: structure-invariant direct methods

Secondary atom site location: difference Fourier map

Hydrogen site location: inferred from neighbouring sites 
H-atom parameters constrained

$w=1 /\left[\sigma^{2}\left(F_{\mathrm{o}}^{2}\right)+(0.0488 P)^{2}+0.2847 P\right]$

where $P=\left(F_{\mathrm{o}}^{2}+2 F_{\mathrm{c}}^{2}\right) / 3$

$(\Delta / \sigma)_{\max }<0.001$

$\Delta \rho_{\max }=0.15$ e $\AA^{-3}$
$\Delta \rho_{\min }=-0.12$ e $\AA^{-3}$

Extinction correction: (SHELXL2018/3;

Sheldrick, 2015),

$\mathrm{Fc}^{*}=\mathrm{kFc}\left[1+0.001 \times \mathrm{xF}^{2} \lambda^{3} / \sin (2 \theta)\right]^{-1 / 4}$

Extinction coefficient: 0.043 (7)

Special details

Geometry. All esds (except the esd in the dihedral angle between two 1.s. planes) are estimated using the full covariance matrix. The cell esds are taken into account individually in the estimation of esds in distances, angles and torsion angles; correlations between esds in cell parameters are only used when they are defined by crystal symmetry. An approximate (isotropic) treatment of cell esds is used for estimating esds involving l.s. planes.

Fractional atomic coordinates and isotropic or equivalent isotropic displacement parameters $\left(\AA^{2}\right)$

\begin{tabular}{lllll}
\hline & $x$ & $y$ & $z$ & $U_{\text {iso }} * U_{\text {eq }}$ \\
\hline N1 & $0.07379(18)$ & $0.10665(19)$ & $0.46684(19)$ & $0.0504(5)$ \\
$\mathrm{N} 2$ & $0.2065(2)$ & $-0.0663(2)$ & $0.2414(2)$ & $0.0657(7)$ \\
$\mathrm{N} 3$ & $0.1836(2)$ & $0.1754(2)$ & $0.8033(2)$ & $0.0699(7)$ \\
$\mathrm{C} 1$ & $0.0393(2)$ & $0.0085(2)$ & $0.3779(2)$ & $0.0465(6)$ \\
$\mathrm{C} 2$ & $0.0345(2)$ & $0.0981(2)$ & $0.5885(2)$ & $0.0476(6)$ \\
$\mathrm{C} 3$ & $0.0864(3)$ & $0.0171(3)$ & $0.2426(2)$ & $0.0576(7)$ \\
$\mathrm{H} 3 \mathrm{~A}$ & 0.009971 & -0.008061 & 0.168567 & $0.069^{*}$ \\
$\mathrm{H} 3 \mathrm{~B}$ & 0.111131 & 0.105746 & 0.226663 & $0.069^{*}$ \\
$\mathrm{C} 4$ & $0.2207(4)$ & $-0.0957(4)$ & $0.1004(3)$ & $0.1046(12)$ \\
$\mathrm{H} 4 \mathrm{~A}$ & 0.234545 & -0.016958 & 0.053752 & $0.157^{*}$ \\
$\mathrm{H} 4 \mathrm{~B}$ & 0.137243 & -0.137698 & 0.052395 & $0.157^{*}$ \\
$\mathrm{H} 4 \mathrm{C}$ & 0.299513 & -0.151713 & 0.102710 & $0.157^{*}$ \\
$\mathrm{C} 5$ & $0.3335(3)$ & $-0.0092(4)$ & $0.3188(3)$ & $0.0979(12)$ \\
$\mathrm{H} 5 \mathrm{C}$ & 0.409862 & -0.068297 & 0.321975 & $0.147^{*}$ \\
$\mathrm{H} 5 \mathrm{~B}$ & 0.322027 & 0.009064 & 0.410892 & $0.147^{*}$ \\
$\mathrm{H} 5 \mathrm{~A}$ & 0.352858 & 0.069624 & 0.275133 & $0.147^{*}$ \\
$\mathrm{C} 6$ & $0.0739(3)$ & $0.2095(2)$ & $0.6863(3)$ & $0.0615(7)$ \\
$\mathrm{H} 6 \mathrm{~A}$ & 0.105348 & 0.281127 & 0.637289 & $0.074^{*}$ \\
H6B & -0.008203 & 0.237656 & 0.719505 & $0.074^{*}$ \\
$\mathrm{C} 7$ & $0.3174(3)$ & $0.1604(4)$ & $0.7623(4)$ & $0.1168(15)$ \\
H7A & 0.346508 & 0.242054 & 0.731787 & $0.175^{*}$ \\
H7B & 0.308288 & 0.098709 & 0.688686 & $0.175^{*}$ \\
H7C & 0.386012 & 0.130348 & 0.839465 & $0.175^{*}$ \\
C8 & $0.1949(4)$ & $0.2720(3)$ & $0.9118(3)$ & $0.1000(12)$ \\
H8A & 0.107852 & 0.277166 & 0.942547 & $0.150^{*}$ \\
H8B & 0.215925 & 0.354560 & 0.876707 & $0.150^{*}$ \\
H8C & 0.268255 & 0.248005 & 0.987590 & $0.150^{*}$ \\
& & & &
\end{tabular}

Atomic displacement parameters $\left(\AA^{2}\right)$

\begin{tabular}{lllllll}
\hline & $U^{11}$ & $U^{22}$ & $U^{33}$ & $U^{12}$ & $U^{13}$ & $U^{23}$ \\
\hline N1 & $0.0471(11)$ & $0.0513(12)$ & $0.0535(12)$ & $0.0003(9)$ & $0.0121(9)$ & $0.0005(10)$ \\
N2 & $0.0654(14)$ & $0.0752(16)$ & $0.0629(14)$ & $0.0117(12)$ & $0.0279(11)$ & $0.0066(12)$ \\
N3 & $0.0630(14)$ & $0.0758(16)$ & $0.0663(14)$ & $-0.0068(12)$ & $0.0021(11)$ & $-0.0189(12)$
\end{tabular}




\begin{tabular}{lllllll} 
C1 & $0.0433(12)$ & $0.0490(14)$ & $0.0465(13)$ & $0.0037(11)$ & $0.0076(10)$ & $0.0006(12)$ \\
C2 & $0.0420(12)$ & $0.0478(14)$ & $0.0522(14)$ & $0.0030(11)$ & $0.0076(10)$ & $-0.0032(11)$ \\
C3 & $0.0597(15)$ & $0.0629(17)$ & $0.0520(14)$ & $0.0059(13)$ & $0.0152(12)$ & $0.0041(12)$ \\
C4 & $0.130(3)$ & $0.117(3)$ & $0.081(2)$ & $0.032(2)$ & $0.056(2)$ & $0.002(2)$ \\
C5 & $0.0565(17)$ & $0.145(3)$ & $0.096(2)$ & $0.001(2)$ & $0.0259(17)$ & $0.019(2)$ \\
C6 & $0.0660(17)$ & $0.0529(15)$ & $0.0666(16)$ & $-0.0012(13)$ & $0.0157(13)$ & $-0.0089(13)$ \\
C7 & $0.060(2)$ & $0.154(4)$ & $0.131(3)$ & $-0.008(2)$ & $0.0089(19)$ & $-0.058(3)$ \\
C8 & $0.105(3)$ & $0.111(3)$ & $0.078(2)$ & $-0.022(2)$ & $0.0064(19)$ & $-0.037(2)$ \\
\hline
\end{tabular}

Geometric parameters $\left(\AA,{ }^{\circ}\right)$

\begin{tabular}{|c|c|c|c|}
\hline $\mathrm{N} 1-\mathrm{C} 2$ & $1.340(3)$ & $\mathrm{C} 4-\mathrm{H} 4 \mathrm{~B}$ & 0.9600 \\
\hline $\mathrm{N} 1-\mathrm{C} 1$ & $1.342(3)$ & $\mathrm{C} 4-\mathrm{H} 4 \mathrm{C}$ & 0.9600 \\
\hline $\mathrm{N} 2-\mathrm{C} 5$ & $1.446(4)$ & $\mathrm{C} 5-\mathrm{H} 5 \mathrm{C}$ & 0.9600 \\
\hline $\mathrm{N} 2-\mathrm{C} 3$ & $1.457(3)$ & $\mathrm{C} 5-\mathrm{H} 5 \mathrm{~B}$ & 0.9600 \\
\hline $\mathrm{N} 2-\mathrm{C} 4$ & $1.463(3)$ & $\mathrm{C} 5-\mathrm{H} 5 \mathrm{~A}$ & 0.9600 \\
\hline $\mathrm{N} 3-\mathrm{C} 7$ & $1.452(4)$ & C6-H6A & 0.9700 \\
\hline N3-C6 & $1.454(3)$ & $\mathrm{C} 6-\mathrm{H} 6 \mathrm{~B}$ & 0.9700 \\
\hline $\mathrm{N} 3-\mathrm{C} 8$ & $1.456(3)$ & C7-H7A & 0.9600 \\
\hline $\mathrm{C} 1-\mathrm{C} 2^{\mathrm{i}}$ & $1.394(3)$ & $\mathrm{C} 7-\mathrm{H} 7 \mathrm{~B}$ & 0.9600 \\
\hline $\mathrm{C} 1-\mathrm{C} 3$ & $1.506(3)$ & $\mathrm{C} 7-\mathrm{H} 7 \mathrm{C}$ & 0.9600 \\
\hline $\mathrm{C} 2-\mathrm{C} 6$ & $1.505(3)$ & $\mathrm{C} 8-\mathrm{H} 8 \mathrm{~A}$ & 0.9600 \\
\hline $\mathrm{C} 3-\mathrm{H} 3 \mathrm{~A}$ & 0.9700 & $\mathrm{C} 8-\mathrm{H} 8 \mathrm{~B}$ & 0.9600 \\
\hline C3-H3B & 0.9700 & $\mathrm{C} 8-\mathrm{H} 8 \mathrm{C}$ & 0.9600 \\
\hline $\mathrm{C} 4-\mathrm{H} 4 \mathrm{~A}$ & 0.9600 & & \\
\hline $\mathrm{C} 2-\mathrm{N} 1-\mathrm{C} 1$ & $117.5(2)$ & $\mathrm{N} 2-\mathrm{C} 5-\mathrm{H} 5 \mathrm{C}$ & 109.5 \\
\hline $\mathrm{C} 5-\mathrm{N} 2-\mathrm{C} 3$ & $111.0(2)$ & $\mathrm{N} 2-\mathrm{C} 5-\mathrm{H} 5 \mathrm{~B}$ & 109.5 \\
\hline $\mathrm{C} 5-\mathrm{N} 2-\mathrm{C} 4$ & $110.8(2)$ & $\mathrm{H} 5 \mathrm{C}-\mathrm{C} 5-\mathrm{H} 5 \mathrm{~B}$ & 109.5 \\
\hline $\mathrm{C} 3-\mathrm{N} 2-\mathrm{C} 4$ & $111.3(2)$ & $\mathrm{N} 2-\mathrm{C} 5-\mathrm{H} 5 \mathrm{~A}$ & 109.5 \\
\hline $\mathrm{C} 7-\mathrm{N} 3-\mathrm{C} 6$ & $111.2(2)$ & $\mathrm{H} 5 \mathrm{C}-\mathrm{C} 5-\mathrm{H} 5 \mathrm{~A}$ & 109.5 \\
\hline $\mathrm{C} 7-\mathrm{N} 3-\mathrm{C} 8$ & $110.0(2)$ & $\mathrm{H} 5 \mathrm{~B}-\mathrm{C} 5-\mathrm{H} 5 \mathrm{~A}$ & 109.5 \\
\hline $\mathrm{C} 6-\mathrm{N} 3-\mathrm{C} 8$ & $110.9(2)$ & $\mathrm{N} 3-\mathrm{C} 6-\mathrm{C} 2$ & $112.3(2)$ \\
\hline $\mathrm{N} 1-\mathrm{C} 1-\mathrm{C} 2^{\mathrm{i}}$ & $121.0(2)$ & $\mathrm{N} 3-\mathrm{C} 6-\mathrm{H} 6 \mathrm{~A}$ & 109.1 \\
\hline $\mathrm{N} 1-\mathrm{C} 1-\mathrm{C} 3$ & $117.3(2)$ & $\mathrm{C} 2-\mathrm{C} 6-\mathrm{H} 6 \mathrm{~A}$ & 109.1 \\
\hline $\mathrm{C} 2 \mathrm{i}-\mathrm{C} 1-\mathrm{C} 3$ & $121.7(2)$ & $\mathrm{N} 3-\mathrm{C} 6-\mathrm{H} 6 \mathrm{~B}$ & 109.1 \\
\hline $\mathrm{N} 1-\mathrm{C} 2-\mathrm{C} 1^{\mathrm{i}}$ & $121.5(2)$ & $\mathrm{C} 2-\mathrm{C} 6-\mathrm{H} 6 \mathrm{~B}$ & 109.1 \\
\hline $\mathrm{N} 1-\mathrm{C} 2-\mathrm{C} 6$ & $116.5(2)$ & $\mathrm{H} 6 \mathrm{~A}-\mathrm{C} 6-\mathrm{H} 6 \mathrm{~B}$ & 107.9 \\
\hline $\mathrm{C} 1-\mathrm{C} 2-\mathrm{C} 6$ & $121.9(2)$ & $\mathrm{N} 3-\mathrm{C} 7-\mathrm{H} 7 \mathrm{~A}$ & 109.5 \\
\hline $\mathrm{N} 2-\mathrm{C} 3-\mathrm{C} 1$ & $111.3(2)$ & $\mathrm{N} 3-\mathrm{C} 7-\mathrm{H} 7 \mathrm{~B}$ & 109.5 \\
\hline $\mathrm{N} 2-\mathrm{C} 3-\mathrm{H} 3 \mathrm{~A}$ & 109.4 & $\mathrm{H} 7 \mathrm{~A}-\mathrm{C} 7-\mathrm{H} 7 \mathrm{~B}$ & 109.5 \\
\hline $\mathrm{C} 1-\mathrm{C} 3-\mathrm{H} 3 \mathrm{~A}$ & 109.4 & $\mathrm{~N} 3-\mathrm{C} 7-\mathrm{H} 7 \mathrm{C}$ & 109.5 \\
\hline $\mathrm{N} 2-\mathrm{C} 3-\mathrm{H} 3 \mathrm{~B}$ & 109.4 & $\mathrm{H} 7 \mathrm{~A}-\mathrm{C} 7-\mathrm{H} 7 \mathrm{C}$ & 109.5 \\
\hline $\mathrm{C} 1-\mathrm{C} 3-\mathrm{H} 3 \mathrm{~B}$ & 109.4 & $\mathrm{H} 7 \mathrm{~B}-\mathrm{C} 7-\mathrm{H} 7 \mathrm{C}$ & 109.5 \\
\hline $\mathrm{H} 3 \mathrm{~A}-\mathrm{C} 3-\mathrm{H} 3 \mathrm{~B}$ & 108.0 & $\mathrm{~N} 3-\mathrm{C} 8-\mathrm{H} 8 \mathrm{~A}$ & 109.5 \\
\hline $\mathrm{N} 2-\mathrm{C} 4-\mathrm{H} 4 \mathrm{~A}$ & 109.5 & $\mathrm{~N} 3-\mathrm{C} 8-\mathrm{H} 8 \mathrm{~B}$ & 109.5 \\
\hline $\mathrm{N} 2-\mathrm{C} 4-\mathrm{H} 4 \mathrm{~B}$ & 109.5 & $\mathrm{H} 8 \mathrm{~A}-\mathrm{C} 8-\mathrm{H} 8 \mathrm{~B}$ & 109.5 \\
\hline $\mathrm{H} 4 \mathrm{~A}-\mathrm{C} 4-\mathrm{H} 4 \mathrm{~B}$ & 109.5 & $\mathrm{~N} 3-\mathrm{C} 8-\mathrm{H} 8 \mathrm{C}$ & 109.5 \\
\hline
\end{tabular}




$\begin{array}{llll}\mathrm{N} 2-\mathrm{C} 4-\mathrm{H} 4 \mathrm{C} & 109.5 & \mathrm{H} 8 \mathrm{~A}-\mathrm{C} 8-\mathrm{H} 8 \mathrm{C} & 109.5 \\ \mathrm{H} 4 \mathrm{~A}-\mathrm{C} 4-\mathrm{H} 4 \mathrm{C} & 109.5 & \mathrm{H} 8 \mathrm{~B}-\mathrm{C} 8-\mathrm{H} 8 \mathrm{C} & 109.5 \\ \mathrm{H} 4 \mathrm{~B}-\mathrm{C} 4-\mathrm{H} 4 \mathrm{C} & 109.5 & & \\ & & & \\ \mathrm{C} 2-\mathrm{N} 1-\mathrm{C} 1-\mathrm{C} 22^{\mathrm{i}} & -0.1(3) & \mathrm{N} 1-\mathrm{C} 1-\mathrm{C} 3-\mathrm{N} 2 & -75.2(3) \\ \mathrm{C} 2-\mathrm{N} 1-\mathrm{C} 1-\mathrm{C} 3 & -178.68(19) & \mathrm{C} 2-\mathrm{C} 1-\mathrm{C} 3-\mathrm{N} 2 & 71.6(3) \\ \mathrm{C} 1-\mathrm{N} 1-\mathrm{C} 2-\mathrm{C} 1^{\mathrm{i}} & 0.1(3) & \mathrm{C} 7-\mathrm{N} 3-\mathrm{C} 6-\mathrm{C} 2 & -165.7(2) \\ \mathrm{C} 1-\mathrm{N} 1-\mathrm{C} 2-\mathrm{C} 6 & -179.79(19) & \mathrm{C} 8-\mathrm{N} 3-\mathrm{C} 6-\mathrm{C} 2 & -108.7(2) \\ \mathrm{C} 5-\mathrm{N} 2-\mathrm{C} 3-\mathrm{C} 1 & -77.0(3) & \mathrm{N} 1-\mathrm{C} 2-\mathrm{C} 6-\mathrm{N} 3 & 71.4(3) \\ \mathrm{C} 4-\mathrm{N} 2-\mathrm{C} 3-\mathrm{C} 1 & 159.1(2) & \mathrm{C} 1-\mathrm{C} 2-\mathrm{C} 6-\mathrm{N} 3 & \end{array}$

Symmetry code: (i) $-x,-y,-z+1$.

Hydrogen-bond geometry $\left(\AA,{ }^{\circ}\right)$

\begin{tabular}{lllll}
\hline$D-\mathrm{H} \cdots A$ & $D-\mathrm{H}$ & $\mathrm{H} \cdots A$ & $D \cdots A$ & $D-\mathrm{H} \cdots A$ \\
\hline $\mathrm{C} 3-\mathrm{H} 3 A \cdots \mathrm{N} 3^{\mathrm{i}}$ & 0.97 & 2.62 & $3.261(4)$ & 124 \\
\hline
\end{tabular}

Symmetry code: (i) $-x,-y,-z+1$.

$N, N^{\prime}, N^{\prime \prime}, N^{\prime \prime \prime}$-[Pyrazine-2,3,5,6\tetrayltetrakis(methylene)] tetrakis( $N$-methylaniline) (II)

Crystal data

$\mathrm{C}_{36} \mathrm{H}_{40} \mathrm{~N}_{6}$

$M_{r}=556.74$

Triclinic, $P \overline{1}$

$a=8.6753(10) \AA$

$b=8.9160(11) \AA$

$c=10.0631(10) \AA$

$\alpha=85.774(10)^{\circ}$

$\beta=73.468(11)^{\circ}$

$\gamma=82.467(11)^{\circ}$

$V=739.21(15) \AA^{3}$

\section{Data collection}

Stoe-Siemens AED2, 4-circle diffractometer

Radiation source: fine-focus sealed tube

Plane graphite monochromator

$\omega / 2 q$ scans

5354 measured reflections

2741 independent reflections

1913 reflections with $I>2 \sigma(I)$

\section{Refinement}

Refinement on $F^{2}$

Least-squares matrix: full

$R\left[F^{2}>2 \sigma\left(F^{2}\right)\right]=0.049$

$w R\left(F^{2}\right)=0.108$

$S=1.12$

2741 reflections

193 parameters

0 restraints
$Z=1$

$F(000)=298$

$D_{\mathrm{x}}=1.251 \mathrm{Mg} \mathrm{m}^{-3}$

Mo $K \alpha$ radiation, $\lambda=0.71073 \AA$

Cell parameters from 28 reflections

$\theta=12.5-17.6^{\circ}$

$\mu=0.08 \mathrm{~mm}^{-1}$

$T=293 \mathrm{~K}$

Block, pale-greenish-yellow

$0.38 \times 0.30 \times 0.27 \mathrm{~mm}$

$$
\begin{aligned}
& R_{\text {int }}=0.031 \\
& \theta_{\max }=25.5^{\circ}, \theta_{\min }=2.1^{\circ} \\
& h=-9 \rightarrow 10 \\
& k=-10 \rightarrow 10 \\
& l=-12 \rightarrow 12
\end{aligned}
$$

2 standard reflections every $60 \mathrm{~min}$ intensity decay: $1 \%$

Primary atom site location: structure-invariant direct methods

Secondary atom site location: difference Fourier map

Hydrogen site location: inferred from neighbouring sites

$\mathrm{H}$-atom parameters constrained

$w=1 /\left[\sigma^{2}\left(F_{\mathrm{o}}{ }^{2}\right)+(0.0254 P)^{2}+0.254 P\right]$

where $P=\left(F_{\mathrm{o}}^{2}+2 F_{\mathrm{c}}^{2}\right) / 3$ 
$(\Delta / \sigma)_{\max }<0.001$

$\Delta \rho_{\max }=0.13 \mathrm{e} \AA^{-3}$

$\Delta \rho_{\min }=-0.14$ e $\AA^{-3}$
Extinction correction: (SHELXL2018/3; Sheldrick 2015),

$\mathrm{Fc}^{*}=\mathrm{kFc}\left[1+0.001 \mathrm{xFc}^{2} \lambda^{3} / \sin (2 \theta)\right]^{-1 / 4}$

Extinction coefficient: $0.026(3)$

Special details

Geometry. All esds (except the esd in the dihedral angle between two 1.s. planes) are estimated using the full covariance matrix. The cell esds are taken into account individually in the estimation of esds in distances, angles and torsion angles; correlations between esds in cell parameters are only used when they are defined by crystal symmetry. An approximate (isotropic) treatment of cell esds is used for estimating esds involving l.s. planes.

Fractional atomic coordinates and isotropic or equivalent isotropic displacement parameters $\left(\AA^{2}\right)$

\begin{tabular}{|c|c|c|c|c|}
\hline & $x$ & $y$ & $z$ & $U_{\text {iso }} * / U_{\text {eq }}$ \\
\hline N1 & $0.46201(19)$ & $0.59971(18)$ & $0.89913(16)$ & 0.0393 \\
\hline $\mathrm{N} 2$ & $0.5739(2)$ & 0.31235 (19) & $0.66461(17)$ & $0.0454(4)$ \\
\hline N3 & $0.2493(2)$ & 0.85914 (19) & $0.95305(17)$ & $0.0481(5)$ \\
\hline $\mathrm{C} 1$ & $0.5585(2)$ & $0.4702(2)$ & $0.86471(19)$ & $0.0366(5)$ \\
\hline $\mathrm{C} 2$ & $0.4027(2)$ & $0.6301(2)$ & $1.0334(2)$ & $0.0378(5)$ \\
\hline $\mathrm{C} 3$ & $0.6246(2)$ & $0.4442(2)$ & $0.7110(2)$ & $0.0437(5)$ \\
\hline $\mathrm{H} 3 \mathrm{~A}$ & 0.590818 & 0.533444 & 0.660508 & $0.052 *$ \\
\hline H3B & 0.741879 & 0.433034 & 0.687257 & $0.052 *$ \\
\hline $\mathrm{C} 4$ & $0.4248(3)$ & $0.3268(2)$ & $0.63526(19)$ & $0.0429(5)$ \\
\hline $\mathrm{C} 5$ & $0.3070(3)$ & $0.4493(3)$ & $0.6795(2)$ & $0.0502(6)$ \\
\hline H5 & 0.328637 & 0.524582 & 0.728604 & $0.060 *$ \\
\hline C6 & $0.1604(3)$ & $0.4603(3)$ & $0.6515(2)$ & $0.0619(7)$ \\
\hline H6 & 0.083983 & 0.542794 & 0.682224 & $0.074 *$ \\
\hline C7 & $0.1237(3)$ & $0.3508(3)$ & $0.5785(3)$ & $0.0674(7)$ \\
\hline $\mathrm{H} 7$ & 0.024139 & 0.359185 & 0.559288 & $0.081 *$ \\
\hline $\mathrm{C} 8$ & $0.2367(3)$ & $0.2306(3)$ & $0.5354(3)$ & $0.0661(7)$ \\
\hline H8 & 0.212966 & 0.155880 & 0.486985 & $0.079^{*}$ \\
\hline C9 & $0.3863(3)$ & $0.2167(2)$ & $0.5618(2)$ & $0.0549(6)$ \\
\hline H9 & 0.461601 & 0.133665 & 0.530481 & $0.066^{*}$ \\
\hline $\mathrm{C} 10$ & $0.7008(3)$ & $0.2013(3)$ & $0.5894(3)$ & $0.0645(7)$ \\
\hline $\mathrm{H} 10 \mathrm{~A}$ & 0.799406 & 0.208832 & 0.612583 & $0.097^{*}$ \\
\hline H10B & 0.717471 & 0.220396 & 0.491434 & $0.097^{*}$ \\
\hline $\mathrm{H} 10 \mathrm{C}$ & 0.669345 & 0.101522 & 0.614401 & $0.097^{*}$ \\
\hline C11 & $0.2938(3)$ & $0.7782(2)$ & $1.0686(2)$ & $0.0488(6)$ \\
\hline H11A & 0.195686 & 0.756971 & 1.138934 & $0.059^{*}$ \\
\hline H11B & 0.348395 & 0.843600 & 1.108603 & $0.059^{*}$ \\
\hline $\mathrm{C} 12$ & $0.1177(2)$ & $0.8292(2)$ & $0.9122(2)$ & $0.0431(5)$ \\
\hline $\mathrm{C} 13$ & $0.0121(3)$ & $0.7258(3)$ & $0.9845(2)$ & $0.0586(6)$ \\
\hline H13 & 0.032849 & 0.671020 & 1.061037 & $0.070^{*}$ \\
\hline C14 & $-0.1220(3)$ & $0.7040(3)$ & $0.9440(3)$ & $0.0697(7)$ \\
\hline H14 & -0.191144 & 0.635993 & 0.994996 & $0.084^{*}$ \\
\hline C15 & $-0.1562(3)$ & $0.7802(3)$ & $0.8302(3)$ & $0.0690(7)$ \\
\hline H15 & -0.247073 & 0.765006 & 0.803564 & $0.083^{*}$ \\
\hline $\mathrm{C} 16$ & $-0.0517(3)$ & $0.8795(3)$ & $0.7571(3)$ & $0.0643(7)$ \\
\hline H16 & -0.072129 & 0.931190 & 0.679059 & $0.077^{*}$ \\
\hline
\end{tabular}




\begin{tabular}{lllll}
$\mathrm{C} 17$ & $0.0822(3)$ & $0.9052(2)$ & $0.7956(2)$ & $0.0516(6)$ \\
$\mathrm{H} 17$ & 0.149960 & 0.973716 & 0.743706 & $0.062^{*}$ \\
$\mathrm{C} 18$ & $0.3667(3)$ & $0.9499(3)$ & $0.8637(3)$ & $0.0633(7)$ \\
$\mathrm{H} 18 \mathrm{~A}$ & 0.407036 & 0.906772 & 0.774033 & $0.095^{*}$ \\
$\mathrm{H} 18 \mathrm{~B}$ & 0.454775 & 0.952213 & 0.903352 & $0.095^{*}$ \\
$\mathrm{H} 18 \mathrm{C}$ & 0.316286 & 1.051147 & 0.854497 & $0.095^{*}$ \\
\hline
\end{tabular}

Atomic displacement parameters $\left(\AA^{2}\right)$

\begin{tabular}{lllllll}
\hline & $U^{11}$ & $U^{22}$ & $U^{33}$ & $U^{12}$ & $U^{13}$ & $U^{23}$ \\
\hline $\mathrm{N} 1$ & $0.0443(10)$ & $0.0408(10)$ & $0.0350(9)$ & $-0.0041(8)$ & $-0.0144(7)$ & $-0.0032(7)$ \\
$\mathrm{N} 2$ & $0.0498(11)$ & $0.0481(10)$ & $0.0390(10)$ & $0.0057(8)$ & $-0.0163(8)$ & $-0.0112(8)$ \\
$\mathrm{N} 3$ & $0.0541(11)$ & $0.0469(11)$ & $0.0455(10)$ & $0.0000(9)$ & $-0.0210(9)$ & $0.0016(8)$ \\
$\mathrm{C} 1$ & $0.0371(11)$ & $0.0405(11)$ & $0.0347(11)$ & $-0.0066(9)$ & $-0.0126(9)$ & $-0.0044(9)$ \\
$\mathrm{C} 2$ & $0.0400(11)$ & $0.0397(11)$ & $0.0366(11)$ & $-0.0044(9)$ & $-0.0147(9)$ & $-0.0040(9)$ \\
$\mathrm{C} 3$ & $0.0454(12)$ & $0.0489(12)$ & $0.0360(11)$ & $-0.0029(10)$ & $-0.0106(9)$ & $-0.0039(9)$ \\
C4 & $0.0523(13)$ & $0.0451(12)$ & $0.0314(11)$ & $-0.0027(10)$ & $-0.0142(9)$ & $0.0021(9)$ \\
C5 & $0.0551(14)$ & $0.0576(14)$ & $0.0379(12)$ & $0.0042(11)$ & $-0.0159(10)$ & $-0.0098(10)$ \\
C6 & $0.0490(14)$ & $0.0793(18)$ & $0.0521(14)$ & $0.0084(13)$ & $-0.0120(11)$ & $-0.0046(13)$ \\
C7 & $0.0535(15)$ & $0.090(2)$ & $0.0637(16)$ & $-0.0169(14)$ & $-0.0239(13)$ & $0.0119(15)$ \\
C8 & $0.0831(19)$ & $0.0582(16)$ & $0.0719(17)$ & $-0.0206(14)$ & $-0.0412(15)$ & $0.0038(13)$ \\
C9 & $0.0747(16)$ & $0.0424(13)$ & $0.0524(14)$ & $-0.0010(11)$ & $-0.0275(12)$ & $-0.0030(10)$ \\
C10 & $0.0631(16)$ & $0.0667(16)$ & $0.0613(15)$ & $0.0207(13)$ & $-0.0210(12)$ & $-0.0223(13)$ \\
C11 & $0.0574(14)$ & $0.0506(13)$ & $0.0406(12)$ & $0.0052(10)$ & $-0.0210(10)$ & $-0.0074(10)$ \\
C12 & $0.0464(12)$ & $0.0381(11)$ & $0.0429(12)$ & $0.0086(9)$ & $-0.0140(10)$ & $-0.0078(9)$ \\
C13 & $0.0673(16)$ & $0.0563(15)$ & $0.0519(14)$ & $-0.0083(12)$ & $-0.0173(12)$ & $0.0048(11)$ \\
C14 & $0.0597(16)$ & $0.0732(18)$ & $0.0723(18)$ & $-0.0179(13)$ & $-0.0071(14)$ & $-0.0057(14)$ \\
C15 & $0.0550(15)$ & $0.0744(18)$ & $0.083(2)$ & $0.0020(14)$ & $-0.0280(14)$ & $-0.0203(15)$ \\
C16 & $0.0704(17)$ & $0.0633(16)$ & $0.0654(16)$ & $0.0085(13)$ & $-0.0356(14)$ & $-0.0040(13)$ \\
C17 & $0.0555(14)$ & $0.0492(13)$ & $0.0509(13)$ & $-0.0012(11)$ & $-0.0196(11)$ & $0.0026(10)$ \\
C18 & $0.0677(16)$ & $0.0617(15)$ & $0.0676(16)$ & $-0.0143(13)$ & $-0.0287(13)$ & $0.0036(13)$ \\
& & & & & &
\end{tabular}

Geometric parameters ( $\left.\AA,{ }^{\circ}\right)$

\begin{tabular}{llll}
\hline $\mathrm{N} 1-\mathrm{C} 2$ & $1.336(2)$ & $\mathrm{C} 8-\mathrm{H} 8$ & 0.9300 \\
$\mathrm{~N} 1-\mathrm{C} 1$ & $1.339(2)$ & $\mathrm{C} 9-\mathrm{H} 9$ & 0.9300 \\
$\mathrm{~N} 2-\mathrm{C} 4$ & $1.395(3)$ & $\mathrm{C} 10-\mathrm{H} 10 \mathrm{~A}$ & 0.9600 \\
$\mathrm{~N} 2-\mathrm{C} 10$ & $1.454(3)$ & $\mathrm{C} 10-\mathrm{H} 10 \mathrm{~B}$ & 0.9600 \\
$\mathrm{~N} 2-\mathrm{C} 3$ & $1.459(3)$ & $\mathrm{C} 10-\mathrm{H} 10 \mathrm{C}$ & 0.9600 \\
$\mathrm{~N} 3-\mathrm{C} 12$ & $1.382(3)$ & $\mathrm{C} 11-\mathrm{H} 11 \mathrm{~A}$ & 0.9700 \\
$\mathrm{~N} 3-\mathrm{C} 11$ & $1.442(2)$ & $\mathrm{C} 11-\mathrm{H} 11 \mathrm{~B}$ & 0.9700 \\
$\mathrm{~N} 3-\mathrm{C} 18$ & $1.445(3)$ & $\mathrm{C} 12-\mathrm{C} 13$ & $1.400(3)$ \\
$\mathrm{C} 1-\mathrm{C} 2^{\mathrm{i}}$ & $1.395(3)$ & $\mathrm{C} 12-\mathrm{C} 17$ & $1.401(3)$ \\
$\mathrm{C} 1-\mathrm{C} 3$ & $1.512(3)$ & $\mathrm{C} 13-\mathrm{C} 14$ & $1.378(3)$ \\
$\mathrm{C} 2-\mathrm{C} 11$ & $1.521(3)$ & $\mathrm{C} 13-\mathrm{H} 13$ & 0.9300 \\
$\mathrm{C} 3-\mathrm{H} 3 \mathrm{~A}$ & 0.9700 & $\mathrm{C} 14-\mathrm{C} 15$ & $1.374(4)$ \\
$\mathrm{C} 3-\mathrm{H} 3 \mathrm{~B}$ & 0.9700 & $\mathrm{C} 14-\mathrm{H} 14$ & 0.9300 \\
$\mathrm{C} 4-\mathrm{C} 9$ & $1.398(3)$ & $\mathrm{C} 15-\mathrm{C} 16$ & $1.371(4)$
\end{tabular}




\begin{tabular}{|c|c|c|c|}
\hline $\mathrm{C} 4-\mathrm{C} 5$ & $1.398(3)$ & $\mathrm{C} 15-\mathrm{H} 15$ & 0.9300 \\
\hline $\mathrm{C} 5-\mathrm{C} 6$ & $1.369(3)$ & $\mathrm{C} 16-\mathrm{C} 17$ & $1.375(3)$ \\
\hline $\mathrm{C} 5-\mathrm{H} 5$ & 0.9300 & $\mathrm{C} 16-\mathrm{H} 16$ & 0.9300 \\
\hline $\mathrm{C} 6-\mathrm{C} 7$ & $1.384(3)$ & $\mathrm{C} 17-\mathrm{H} 17$ & 0.9300 \\
\hline $\mathrm{C} 6-\mathrm{H} 6$ & 0.9300 & $\mathrm{C} 18-\mathrm{H} 18 \mathrm{~A}$ & 0.9600 \\
\hline $\mathrm{C} 7-\mathrm{C} 8$ & $1.359(4)$ & $\mathrm{C} 18-\mathrm{H} 18 \mathrm{~B}$ & 0.9600 \\
\hline $\mathrm{C} 7-\mathrm{H} 7$ & 0.9300 & $\mathrm{C} 18-\mathrm{H} 18 \mathrm{C}$ & 0.9600 \\
\hline $\mathrm{C} 8-\mathrm{C} 9$ & $1.386(3)$ & & \\
\hline $\mathrm{C} 2-\mathrm{N} 1-\mathrm{C} 1$ & $118.46(16)$ & $\mathrm{C} 7-\mathrm{C} 8-\mathrm{C} 9$ & $121.7(2)$ \\
\hline $\mathrm{C} 4-\mathrm{N} 2-\mathrm{C} 10$ & $117.64(17)$ & $\mathrm{C} 7-\mathrm{C} 8-\mathrm{H} 8$ & 119.2 \\
\hline $\mathrm{C} 4-\mathrm{N} 2-\mathrm{C} 3$ & $118.71(16)$ & $\mathrm{C} 9-\mathrm{C} 8-\mathrm{H} 8$ & 119.2 \\
\hline $\mathrm{C} 10-\mathrm{N} 2-\mathrm{C} 3$ & $117.17(18)$ & $\mathrm{C} 8-\mathrm{C} 9-\mathrm{C} 4$ & $120.4(2)$ \\
\hline $\mathrm{C} 12-\mathrm{N} 3-\mathrm{C} 11$ & $122.00(18)$ & $\mathrm{C} 8-\mathrm{C} 9-\mathrm{H} 9$ & 119.8 \\
\hline $\mathrm{C} 12-\mathrm{N} 3-\mathrm{C} 18$ & $120.07(17)$ & $\mathrm{C} 4-\mathrm{C} 9-\mathrm{H} 9$ & 119.8 \\
\hline $\mathrm{C} 11-\mathrm{N} 3-\mathrm{C} 18$ & $116.70(18)$ & $\mathrm{N} 3-\mathrm{C} 12-\mathrm{C} 13$ & $122.77(19)$ \\
\hline $\mathrm{N} 1-\mathrm{C} 1-\mathrm{C} 2^{\mathrm{i}}$ & $120.79(16)$ & $\mathrm{N} 3-\mathrm{C} 12-\mathrm{C} 17$ & $120.5(2)$ \\
\hline $\mathrm{N} 1-\mathrm{C} 1-\mathrm{C} 3$ & $115.85(16)$ & $\mathrm{C} 13-\mathrm{C} 12-\mathrm{C} 17$ & $116.7(2)$ \\
\hline $\mathrm{C} 2{ }^{\mathrm{i}}-\mathrm{C} 1-\mathrm{C} 3$ & $123.33(17)$ & $\mathrm{C} 14-\mathrm{C} 13-\mathrm{C} 12$ & $121.0(2)$ \\
\hline $\mathrm{N} 1-\mathrm{C} 2-\mathrm{C} 1^{\mathrm{i}}$ & $120.74(17)$ & $\mathrm{C} 14-\mathrm{C} 13-\mathrm{H} 13$ & 119.5 \\
\hline $\mathrm{N} 1-\mathrm{C} 2-\mathrm{C} 11$ & $117.03(17)$ & $\mathrm{C} 12-\mathrm{C} 13-\mathrm{H} 13$ & 119.5 \\
\hline $\mathrm{C} 1-\mathrm{C} 2-\mathrm{C} 11$ & $122.23(17)$ & $\mathrm{C} 15-\mathrm{C} 14-\mathrm{C} 13$ & $121.6(2)$ \\
\hline $\mathrm{N} 2-\mathrm{C} 3-\mathrm{C} 1$ & $114.84(17)$ & $\mathrm{C} 15-\mathrm{C} 14-\mathrm{H} 14$ & 119.2 \\
\hline $\mathrm{N} 2-\mathrm{C} 3-\mathrm{H} 3 \mathrm{~A}$ & 108.6 & $\mathrm{C} 13-\mathrm{C} 14-\mathrm{H} 14$ & 119.2 \\
\hline $\mathrm{C} 1-\mathrm{C} 3-\mathrm{H} 3 \mathrm{~A}$ & 108.6 & $\mathrm{C} 16-\mathrm{C} 15-\mathrm{C} 14$ & $117.7(2)$ \\
\hline $\mathrm{N} 2-\mathrm{C} 3-\mathrm{H} 3 \mathrm{~B}$ & 108.6 & $\mathrm{C} 16-\mathrm{C} 15-\mathrm{H} 15$ & 121.1 \\
\hline $\mathrm{C} 1-\mathrm{C} 3-\mathrm{H} 3 \mathrm{~B}$ & 108.6 & $\mathrm{C} 14-\mathrm{C} 15-\mathrm{H} 15$ & 121.1 \\
\hline $\mathrm{H} 3 \mathrm{~A}-\mathrm{C} 3-\mathrm{H} 3 \mathrm{~B}$ & 107.5 & $\mathrm{C} 15-\mathrm{C} 16-\mathrm{C} 17$ & $122.1(2)$ \\
\hline $\mathrm{N} 3-\mathrm{C} 11-\mathrm{C} 2$ & $115.02(17)$ & $\mathrm{C} 15-\mathrm{C} 16-\mathrm{H} 16$ & 119.0 \\
\hline $\mathrm{N} 3-\mathrm{C} 11-\mathrm{H} 11 \mathrm{~A}$ & 108.5 & $\mathrm{C} 17-\mathrm{C} 16-\mathrm{H} 16$ & 119.0 \\
\hline $\mathrm{C} 2-\mathrm{C} 11-\mathrm{H} 11 \mathrm{~A}$ & 108.5 & $\mathrm{C} 16-\mathrm{C} 17-\mathrm{C} 12$ & $120.8(2)$ \\
\hline $\mathrm{N} 3-\mathrm{C} 11-\mathrm{H} 11 \mathrm{~B}$ & 108.5 & $\mathrm{C} 16-\mathrm{C} 17-\mathrm{H} 17$ & 119.6 \\
\hline $\mathrm{C} 2-\mathrm{C} 11-\mathrm{H} 11 \mathrm{~B}$ & 108.5 & $\mathrm{C} 12-\mathrm{C} 17-\mathrm{H} 17$ & 119.6 \\
\hline $\mathrm{H} 11 \mathrm{~A}-\mathrm{C} 11-\mathrm{H} 11 \mathrm{~B}$ & 107.5 & $\mathrm{~N} 3-\mathrm{C} 18-\mathrm{H} 18 \mathrm{~A}$ & 109.5 \\
\hline $\mathrm{N} 2-\mathrm{C} 4-\mathrm{C} 9$ & $120.81(19)$ & $\mathrm{N} 3-\mathrm{C} 18-\mathrm{H} 18 \mathrm{~B}$ & 109.5 \\
\hline $\mathrm{N} 2-\mathrm{C} 4-\mathrm{C} 5$ & $121.91(19)$ & $\mathrm{H} 18 \mathrm{~A}-\mathrm{C} 18-\mathrm{H} 18 \mathrm{~B}$ & 109.5 \\
\hline $\mathrm{C} 9-\mathrm{C} 4-\mathrm{C} 5$ & $117.3(2)$ & $\mathrm{N} 3-\mathrm{C} 18-\mathrm{H} 18 \mathrm{C}$ & 109.5 \\
\hline $\mathrm{C} 6-\mathrm{C} 5-\mathrm{C} 4$ & $121.1(2)$ & $\mathrm{H} 18 \mathrm{~A}-\mathrm{C} 18-\mathrm{H} 18 \mathrm{C}$ & 109.5 \\
\hline $\mathrm{C} 6-\mathrm{C} 5-\mathrm{H} 5$ & 119.5 & $\mathrm{H} 18 \mathrm{~B}-\mathrm{C} 18-\mathrm{H} 18 \mathrm{C}$ & 109.5 \\
\hline $\mathrm{C} 4-\mathrm{C} 5-\mathrm{H} 5$ & 119.5 & $\mathrm{~N} 2-\mathrm{C} 10-\mathrm{H} 10 \mathrm{~A}$ & 109.5 \\
\hline $\mathrm{C} 5-\mathrm{C} 6-\mathrm{C} 7$ & $121.2(2)$ & $\mathrm{N} 2-\mathrm{C} 10-\mathrm{H} 10 \mathrm{~B}$ & 109.5 \\
\hline $\mathrm{C} 5-\mathrm{C} 6-\mathrm{H} 6$ & 119.4 & $\mathrm{H} 10 \mathrm{~A}-\mathrm{C} 10-\mathrm{H} 10 \mathrm{~B}$ & 109.5 \\
\hline $\mathrm{C} 7-\mathrm{C} 6-\mathrm{H} 6$ & 119.4 & $\mathrm{~N} 2-\mathrm{C} 10-\mathrm{H} 10 \mathrm{C}$ & 109.5 \\
\hline $\mathrm{C} 8-\mathrm{C} 7-\mathrm{C} 6$ & $118.4(2)$ & $\mathrm{H} 10 \mathrm{~A}-\mathrm{C} 10-\mathrm{H} 10 \mathrm{C}$ & 109.5 \\
\hline $\mathrm{C} 8-\mathrm{C} 7-\mathrm{H} 7$ & 120.8 & $\mathrm{H} 10 \mathrm{~B}-\mathrm{C} 10-\mathrm{H} 10 \mathrm{C}$ & 109.5 \\
\hline $\mathrm{C} 6-\mathrm{C} 7-\mathrm{H} 7$ & 120.8 & & \\
\hline $\mathrm{C} 2-\mathrm{N} 1-\mathrm{C} 1-\mathrm{C} 2^{\mathrm{i}}$ & $-0.5(3)$ & $\mathrm{C} 4-\mathrm{C} 5-\mathrm{C} 6-\mathrm{C} 7$ & $-0.1(4)$ \\
\hline
\end{tabular}




$\begin{array}{llll}\mathrm{C} 2-\mathrm{N} 1-\mathrm{C} 1-\mathrm{C} 3 & -178.66(17) & \mathrm{C} 5-\mathrm{C} 6-\mathrm{C} 7-\mathrm{C} 8 & 0.4(4) \\ \mathrm{C} 1-\mathrm{N} 1-\mathrm{C} 2-\mathrm{C} 1{ }^{\mathrm{i}} & 0.5(3) & \mathrm{C} 6-\mathrm{C} 7-\mathrm{C} 8-\mathrm{C} 9 & -0.6(4) \\ \mathrm{C} 1-\mathrm{N} 1-\mathrm{C} 2-\mathrm{C} 11 & 179.92(17) & \mathrm{C} 7-\mathrm{C} 8-\mathrm{C} 9-\mathrm{C} 4 & 0.5(4) \\ \mathrm{C} 4-\mathrm{N} 2-\mathrm{C} 3-\mathrm{C} 1 & 83.8(2) & \mathrm{N} 2-\mathrm{C} 4-\mathrm{C} 9-\mathrm{C} 8 & 178.8(2) \\ \mathrm{C} 10-\mathrm{N} 2-\mathrm{C} 3-\mathrm{C} 1 & -125.0(2) & \mathrm{C} 5-\mathrm{C} 4-\mathrm{C} 9-\mathrm{C} 8 & -0.2(3) \\ \mathrm{N} 1-\mathrm{C} 1-\mathrm{C} 3-\mathrm{N} 2 & -117.34(19) & \mathrm{C} 11-\mathrm{N} 3-\mathrm{C} 12-\mathrm{C} 13 & 4.2(3) \\ \mathrm{C} 2-\mathrm{C} 1-\mathrm{C} 3-\mathrm{N} 2 & 64.5(2) & \mathrm{C} 18-\mathrm{N} 3-\mathrm{C} 12-\mathrm{C} 13 & 171.1(2) \\ \mathrm{C} 12-\mathrm{N} 3-\mathrm{C} 11-\mathrm{C} 2 & 86.2(2) & \mathrm{C} 11-\mathrm{N} 3-\mathrm{C} 12-\mathrm{C} 17 & -177.13(19) \\ \mathrm{C} 18-\mathrm{N} 3-\mathrm{C} 11-\mathrm{C} 2 & -81.1(2) & \mathrm{C} 18-\mathrm{N} 3-\mathrm{C} 12-\mathrm{C} 17 & -10.2(3) \\ \mathrm{N} 1-\mathrm{C} 2-\mathrm{C} 11-\mathrm{N} 3 & 8.2(3) & \mathrm{N} 3-\mathrm{C} 12-\mathrm{C} 13-\mathrm{C} 14 & 177.1(2) \\ \mathrm{C} 1-\mathrm{C} 2-\mathrm{C} 11-\mathrm{N} 3 & -172.42(18) & \mathrm{C} 17-\mathrm{C} 12-\mathrm{C} 13-\mathrm{C} 14 & -1.7(3) \\ \mathrm{C} 10-\mathrm{N} 2-\mathrm{C} 4-\mathrm{C} 9 & 14.5(3) & \mathrm{C} 12-\mathrm{C} 13-\mathrm{C} 14-\mathrm{C} 15 & 1.2(4) \\ \mathrm{C} 3-\mathrm{N} 2-\mathrm{C} 4-\mathrm{C} 9 & 165.50(19) & \mathrm{C} 13-\mathrm{C} 14-\mathrm{C} 15-\mathrm{C} 16 & 0.1(4) \\ \mathrm{C} 10-\mathrm{N} 2-\mathrm{C} 4-\mathrm{C} 5 & -166.6(2) & \mathrm{C} 14-\mathrm{C} 15-\mathrm{C} 16-\mathrm{C} 17 & -0.8(4) \\ \mathrm{C} 3-\mathrm{N} 2-\mathrm{C} 4-\mathrm{C} 5 & -15.6(3) & \mathrm{C} 15-\mathrm{C} 16-\mathrm{C} 17-\mathrm{C} 12 & 0.3(4) \\ \mathrm{N} 2-\mathrm{C} 4-\mathrm{C} 5-\mathrm{C} 6 & -178.9(2) & \mathrm{N} 3-\mathrm{C} 12-\mathrm{C} 17-\mathrm{C} 16 & -177.8(2) \\ \mathrm{C} 9-\mathrm{C} 4-\mathrm{C} 5-\mathrm{C} 6 & 0.0(3) & \mathrm{C} 13-\mathrm{C} 12-\mathrm{C} 17-\mathrm{C} 16 & 1.0(3) \\ \end{array}$

Symmetry code: (i) $-x+1,-y+1,-z+2$.

Hydrogen-bond geometry $\left(\AA,{ }^{\circ}\right)$

$\mathrm{Cg} 2$ and $\mathrm{Cg} 3$ are the centroids of rings $\mathrm{C} 4-\mathrm{C} 9$ and $\mathrm{C} 12-\mathrm{C} 17$, respectively.

\begin{tabular}{lllll}
\hline$D-\mathrm{H} \cdots A$ & $D-\mathrm{H}$ & $\mathrm{H} \cdots A$ & $D \cdots A$ & $D-\mathrm{H} \cdots A$ \\
\hline $\mathrm{C} 5-\mathrm{H} 5 \cdots \mathrm{N} 1$ & 0.93 & 2.50 & $3.331(3)$ & 149 \\
$\mathrm{C} 6-\mathrm{H} 6 \cdots C g 3$ & 0.93 & 2.99 & $3.804(3)$ & 147 \\
$\mathrm{C} 3-\mathrm{H} 3 A \cdots \mathrm{Cg} 2^{\mathrm{ii}}$ & 0.97 & 2.83 & $3.561(2)$ & 133 \\
\hline
\end{tabular}

Symmetry code: (ii) $-x+1,-y+1,-z+1$.

N,N'-[(6-Phenyl-6,7-dihydro-5H-pyrrolo[3,4-\b]pyrazine-2,3-diyl)bis(methylene)]bis( $N$-methylaniline) (III)

Crystal data

$\mathrm{C}_{28} \mathrm{H}_{29} \mathrm{~N}_{5}$

$M_{r}=435.56$

Triclinic, $P \overline{1}$

$a=8.686(1) \AA$

$b=9.7731(11) \AA$

$c=14.3948(16) \AA$

$\alpha=85.915(13)^{\circ}$

$\beta=75.349(13)^{\circ}$

$\gamma=78.891(13)^{\circ}$

$V=1159.8(2) \AA^{3}$

\section{Data collection}

Stoe IPDS 1

diffractometer

Radiation source: fine-focus sealed tube

Plane graphite monochromator

$\varphi$ rotation scans

8653 measured reflections

3953 independent reflections
$Z=2$

$F(000)=464$

$D_{\mathrm{x}}=1.247 \mathrm{Mg} \mathrm{m}^{-3}$

Mo $K \alpha$ radiation, $\lambda=0.71073 \AA$

Cell parameters from 5000 reflections

$\theta=1.7-26.1^{\circ}$

$\mu=0.08 \mathrm{~mm}^{-1}$

$T=293 \mathrm{~K}$

Hexagonal plate, pale yellow

$0.45 \times 0.35 \times 0.10 \mathrm{~mm}$

1518 reflections with $I>2 \sigma(I)$

$R_{\text {int }}=0.051$

$\theta_{\text {max }}=25.3^{\circ}, \theta_{\text {min }}=2.1^{\circ}$

$h=-10 \rightarrow 10$

$k=-11 \rightarrow 11$

$l=-17 \rightarrow 17$ 


\section{Refinement}

Refinement on $F^{2}$

Least-squares matrix: full

$R\left[F^{2}>2 \sigma\left(F^{2}\right)\right]=0.035$

$w R\left(F^{2}\right)=0.079$

$S=0.68$

3953 reflections

301 parameters

0 restraints

Primary atom site location: structure-invariant direct methods

Secondary atom site location: difference Fourier map
Hydrogen site location: inferred from neighbouring sites

$\mathrm{H}$-atom parameters constrained

$w=1 /\left[\sigma^{2}\left(F_{\mathrm{o}}^{2}\right)+(0.0308 P)^{2}\right]$

where $P=\left(F_{\mathrm{o}}^{2}+2 F_{\mathrm{c}}{ }^{2}\right) / 3$

$(\Delta / \sigma)_{\max }<0.001$

$\Delta \rho_{\max }=0.12 \mathrm{e} \AA^{-3}$

$\Delta \rho_{\text {min }}=-0.12$ e $\AA^{-3}$

Extinction correction: (SHELXL2018/3; Sheldrick, 2015), $\mathrm{Fc}^{*}=\mathrm{kFc}\left[1+0.001 \times \mathrm{Fc}^{2} \lambda^{3} / \sin (2 \theta)\right]^{-1 / 4}$

Extinction coefficient: 0.0154 (11)

\section{Special details}

Geometry. All esds (except the esd in the dihedral angle between two 1.s. planes) are estimated using the full covariance matrix. The cell esds are taken into account individually in the estimation of esds in distances, angles and torsion angles; correlations between esds in cell parameters are only used when they are defined by crystal symmetry. An approximate (isotropic) treatment of cell esds is used for estimating esds involving l.s. planes.

Fractional atomic coordinates and isotropic or equivalent isotropic displacement parameters $\left(\AA^{2}\right)$

\begin{tabular}{|c|c|c|c|c|}
\hline & $x$ & $y$ & $z$ & $U_{\text {iso }} * / U_{\text {eq }}$ \\
\hline N1 & $0.4060(2)$ & $0.8786(2)$ & $0.20916(11)$ & $0.0559(5)$ \\
\hline N2 & $0.2666(2)$ & $0.9790(2)$ & $0.05437(12)$ & $0.0596(6)$ \\
\hline N3 & $0.5915(2)$ & $0.6863(2)$ & $-0.00687(11)$ & $0.0641(6)$ \\
\hline N4 & $-0.0765(2)$ & $1.1354(2)$ & 0.19845 (12) & $0.0567(5)$ \\
\hline N5 & $0.2256(2)$ & $0.9508(2)$ & $0.39598(12)$ & $0.0607(6)$ \\
\hline $\mathrm{C} 1$ & $0.2797(3)$ & $0.9865(2)$ & $0.21849(14)$ & $0.0536(6)$ \\
\hline $\mathrm{C} 2$ & $0.4566(2)$ & $0.8223(2)$ & $0.12258(14)$ & $0.0510(6)$ \\
\hline $\mathrm{C} 3$ & $0.3862(3)$ & $0.8699(3)$ & $0.04787(13)$ & $0.0522(6)$ \\
\hline $\mathrm{C} 4$ & $0.2133(2)$ & $1.0385(2)$ & $0.14142(15)$ & $0.0550(6)$ \\
\hline $\mathrm{C} 5$ & $0.5911(3)$ & $0.7029(2)$ & $0.09341(13)$ & $0.0611(7)$ \\
\hline H5A & 0.692953 & 0.724226 & 0.098842 & $0.073 *$ \\
\hline H5B & 0.570113 & 0.619743 & 0.131738 & $0.073 *$ \\
\hline C6 & $0.4643(2)$ & $0.7825(2)$ & $-0.03875(13)$ & $0.0577(7)$ \\
\hline H6A & 0.388274 & 0.733319 & -0.055210 & $0.069 *$ \\
\hline H6B & 0.508592 & 0.838726 & -0.093682 & $0.069 *$ \\
\hline $\mathrm{C} 7$ & $0.6870(3)$ & 0.5773 & $-0.06142(15)$ & $0.0559(6)$ \\
\hline $\mathrm{C} 8$ & $0.8004(3)$ & $0.4812(3)$ & $-0.02614(15)$ & $0.0620(7)$ \\
\hline H8 & 0.810894 & 0.489435 & 0.035832 & $0.074 *$ \\
\hline C9 & $0.8976(3)$ & $0.3735(3)$ & $-0.08282(17)$ & $0.0725(8)$ \\
\hline H9 & 0.972506 & 0.309893 & -0.058215 & $0.087 *$ \\
\hline $\mathrm{C} 10$ & $0.8853(3)$ & $0.3590(3)$ & $-0.17452(19)$ & $0.0773(8)$ \\
\hline $\mathrm{H} 10$ & 0.951563 & 0.286567 & -0.212066 & $0.093 *$ \\
\hline C11 & $0.7738(3)$ & $0.4528(3)$ & $-0.21026(16)$ & $0.0764(8)$ \\
\hline H11 & 0.764776 & 0.443527 & -0.272410 & $0.092 *$ \\
\hline $\mathrm{C} 12$ & $0.6751(3)$ & $0.5608(3)$ & $-0.15511(15)$ & $0.0665(7)$ \\
\hline H12 & 0.599899 & 0.623164 & -0.180367 & $0.080 *$ \\
\hline $\mathrm{C} 13$ & $0.2210(3)$ & $1.0516(2)$ & $0.31746(14)$ & $0.0602(7)$ \\
\hline
\end{tabular}




\begin{tabular}{|c|c|c|c|c|}
\hline H13A & 0.287658 & 1.119063 & 0.321236 & $0.072 *$ \\
\hline H13B & 0.110758 & 1.101454 & 0.324780 & $0.072 *$ \\
\hline $\mathrm{C} 14$ & $0.1121(3)$ & $0.8654(3)$ & $0.42226(14)$ & $0.0560(6)$ \\
\hline $\mathrm{C} 15$ & $-0.0211(3)$ & $0.8838(3)$ & $0.38231(15)$ & $0.0629(7)$ \\
\hline H15 & -0.032502 & 0.953551 & 0.335842 & $0.076^{*}$ \\
\hline $\mathrm{C} 16$ & $-0.1360(3)$ & $0.8000(3)$ & $0.41067(19)$ & $0.0781(8)$ \\
\hline H16 & -0.224404 & 0.814656 & 0.383465 & $0.094 *$ \\
\hline $\mathrm{C} 17$ & -0.1225 & $0.6956(3)$ & $0.4781(2)$ & $0.0906(9)$ \\
\hline $\mathrm{H} 17$ & -0.199630 & 0.638472 & 0.496365 & $0.109^{*}$ \\
\hline $\mathrm{C} 18$ & $0.0080(5)$ & $0.6771(3)$ & $0.51850(19)$ & $0.0919(10)$ \\
\hline H18 & 0.017561 & 0.607346 & 0.565218 & $0.110^{*}$ \\
\hline C19 & $0.1240(3)$ & $0.7587(3)$ & $0.49158(17)$ & $0.0713(8)$ \\
\hline H19 & 0.211426 & 0.743206 & 0.519603 & $0.086^{*}$ \\
\hline $\mathrm{C} 20$ & 0.3718 & $0.9195(3)$ & $0.43102(17)$ & $0.0861(9)$ \\
\hline $\mathrm{H} 20 \mathrm{~A}$ & 0.435685 & 0.990627 & 0.408568 & $0.129 *$ \\
\hline H20B & 0.433210 & 0.830763 & 0.407665 & $0.129 *$ \\
\hline $\mathrm{H} 20 \mathrm{C}$ & 0.343173 & 0.916359 & 0.499973 & $0.129 *$ \\
\hline $\mathrm{C} 21$ & $0.0812(3)$ & $1.1664(2)$ & $0.14886(15)$ & $0.0651(7)$ \\
\hline $\mathrm{H} 21 \mathrm{~A}$ & 0.106550 & 1.237790 & 0.183089 & $0.078 *$ \\
\hline $\mathrm{H} 21 \mathrm{~B}$ & 0.077662 & 1.202914 & 0.084840 & $0.078^{*}$ \\
\hline $\mathrm{C} 22$ & $-0.1951(3)$ & $1.2457(3)$ & $0.24527(14)$ & $0.0534(6)$ \\
\hline $\mathrm{C} 23$ & $-0.1678(3)$ & $1.3796(3)$ & $0.24765(15)$ & $0.0688(7)$ \\
\hline $\mathrm{H} 23$ & -0.069134 & 1.402028 & 0.213889 & $0.083^{*}$ \\
\hline $\mathrm{C} 24$ & -0.2859 (4) & $1.4813(3)$ & $0.29992(19)$ & $0.0860(9)$ \\
\hline $\mathrm{H} 24$ & -0.264807 & 1.570861 & 0.301192 & $0.103^{*}$ \\
\hline $\mathrm{C} 25$ & $-0.4332(4)$ & $1.4517(4)$ & $0.34971(19)$ & $0.0920(11)$ \\
\hline $\mathrm{H} 25$ & -0.511567 & 1.519698 & 0.385288 & $0.110^{*}$ \\
\hline $\mathrm{C} 26$ & $-0.4620(3)$ & $1.3199(4)$ & $0.34573(18)$ & $0.0911(10)$ \\
\hline $\mathrm{H} 26$ & -0.562113 & 1.299000 & 0.378099 & $0.109^{*}$ \\
\hline $\mathrm{C} 27$ & $-0.3461(3)$ & $1.2173(3)$ & $0.29485(16)$ & $0.0719(8)$ \\
\hline $\mathrm{H} 27$ & -0.368766 & 1.128393 & 0.293559 & $0.086^{*}$ \\
\hline $\mathrm{C} 28$ & $-0.1287(3)$ & $1.0303(3)$ & $0.15362(17)$ & $0.0805(8)$ \\
\hline $\mathrm{H} 28 \mathrm{~A}$ & -0.200292 & 0.983359 & 0.201593 & $0.121^{*}$ \\
\hline H28B & -0.036112 & 0.963950 & 0.123096 & $0.121^{*}$ \\
\hline $\mathrm{H} 28 \mathrm{C}$ & -0.184352 & 1.073996 & 0.106462 & $0.121 *$ \\
\hline
\end{tabular}

Atomic displacement parameters $\left(\AA^{2}\right)$

\begin{tabular}{lllllll}
\hline & $U^{11}$ & $U^{22}$ & $U^{33}$ & $U^{12}$ & $U^{13}$ & $U^{23}$ \\
\hline N1 & $0.0524(11)$ & $0.0660(15)$ & $0.0465(11)$ & $-0.0088(11)$ & $-0.0077(9)$ & $-0.0042(9)$ \\
N2 & $0.0533(12)$ & $0.0728(15)$ & $0.0465(11)$ & $-0.0058(11)$ & $-0.0064(9)$ & $0.0024(10)$ \\
N3 & $0.0620(12)$ & $0.0785(16)$ & $0.0446(11)$ & $0.0103(12)$ & $-0.0146(9)$ & $-0.0111(10)$ \\
N4 & $0.0522(12)$ & $0.0551(14)$ & $0.0605(11)$ & $-0.0069(11)$ & $-0.0106(10)$ & $-0.0064(10)$ \\
N5 & $0.0562(13)$ & $0.0813(17)$ & $0.0459(11)$ & $-0.0163(12)$ & $-0.0104(10)$ & $-0.0081(10)$ \\
C1 & $0.0503(14)$ & $0.0603(18)$ & $0.0468(13)$ & $-0.0137(13)$ & $-0.0020(11)$ & $-0.0047(11)$ \\
C2 & $0.0461(13)$ & $0.0629(17)$ & $0.0413(13)$ & $-0.0085(13)$ & $-0.0070(11)$ & $-0.0013(12)$ \\
C3 & $0.0469(13)$ & $0.0650(17)$ & $0.0419(13)$ & $-0.0106(13)$ & $-0.0056(11)$ & $0.0000(12)$ \\
C4 & $0.0498(14)$ & $0.0601(17)$ & $0.0505(14)$ & $-0.0082(13)$ & $-0.0063(12)$ & $0.0022(12)$
\end{tabular}




$\begin{array}{lllllll}\text { C5 } & 0.0567(14) & 0.0749(19) & 0.0467(13) & -0.0009(14) & -0.0100(11) & -0.0089(12) \\ \text { C6 } & 0.0524(14) & 0.0736(18) & 0.0449(12) & -0.0084(13) & -0.0094(11) & -0.0039(12) \\ \text { C7 } & 0.0486(14) & 0.0627(18) & 0.0532(14) & -0.0095(14) & -0.0052(11) & -0.0076(12) \\ \text { C8 } & 0.0555(14) & 0.0709(19) & 0.0563(14) & -0.0066(15) & -0.0098(12) & -0.0068(13) \\ \text { C9 } & 0.0645(17) & 0.069(2) & 0.0777(18) & -0.0034(15) & -0.0112(14) & -0.0085(15) \\ \text { C10 } & 0.0739(19) & 0.074(2) & 0.0766(18) & -0.0108(17) & 0.0001(15) & -0.0247(15) \\ \text { C11 } & 0.0800(19) & 0.088(2) & 0.0597(15) & -0.0135(18) & -0.0103(14) & -0.0186(15) \\ \text { C12 } & 0.0623(16) & 0.080(2) & 0.0562(15) & -0.0069(15) & -0.0135(12) & -0.0119(13) \\ \text { C13 } & 0.0618(16) & 0.0653(18) & 0.0513(13) & -0.0143(13) & -0.0053(11) & -0.0109(13) \\ \text { C14 } & 0.0589(16) & 0.0647(19) & 0.0397(13) & -0.0072(15) & -0.0032(12) & -0.0143(12) \\ \text { C15 } & 0.0598(16) & 0.074(2) & 0.0540(14) & -0.0175(15) & -0.0057(13) & -0.0109(13) \\ \text { C16 } & 0.070(2) & 0.085(2) & 0.0761(18) & -0.0172(18) & -0.0039(15) & -0.0237(17) \\ \text { C17 } & 0.092(2) & 0.079(3) & 0.092(2) & -0.039(2) & 0.0180(18) & -0.0243(19) \\ \text { C18 } & 0.127(3) & 0.069(2) & 0.0706(19) & -0.018(2) & -0.006(2) & -0.0067(15) \\ \text { C19 } & 0.086(2) & 0.064(2) & 0.0615(16) & -0.0086(17) & -0.0154(14) & -0.0066(14) \\ \text { C20 } & 0.0670(17) & 0.122(3) & 0.0789(17) & -0.0167(17) & -0.0316(14) & -0.0145(16) \\ \text { C21 } & 0.0603(16) & 0.0617(19) & 0.0639(14) & -0.0040(14) & -0.0043(12) & 0.0023(13) \\ \text { C22 } & 0.0536(16) & 0.0608(19) & 0.0442(12) & -0.0012(14) & -0.0162(11) & 0.0003(12) \\ \text { C23 } & 0.0726(17) & 0.061(2) & 0.0673(16) & -0.0041(17) & -0.0117(13) & -0.0061(14) \\ \text { C24 } & 0.100(2) & 0.065(2) & 0.0893(19) & 0.0040(19) & -0.0269(18) & -0.0202(16) \\ \text { C25 } & 0.081(2) & 0.110(3) & 0.0742(19) & 0.019(2) & -0.0162(17) & -0.0368(19) \\ \text { C26 } & 0.0629(18) & 0.121(3) & 0.0784(19) & 0.003(2) & -0.0059(14) & -0.0256(19) \\ \text { C27 } & 0.0598(16) & 0.080(2) & 0.0711(15) & -0.0083(16) & -0.0100(14) & -0.0066(14) \\ \text { C28 } & 0.0687(17) & 0.086(2) & 0.0927(18) & -0.0024(16) & -0.0318(15) & -0.0313(16) \\ & & & & & & \end{array}$

Geometric parameters $\left(\AA,{ }^{\circ}\right)$

\begin{tabular}{llll}
\hline $\mathrm{N} 1-\mathrm{C} 2$ & $1.333(2)$ & $\mathrm{C} 12-\mathrm{H} 12$ & 0.9300 \\
$\mathrm{~N} 1-\mathrm{C} 1$ & $1.354(2)$ & $\mathrm{C} 13-\mathrm{H} 13 \mathrm{~A}$ & 0.9700 \\
$\mathrm{~N} 2-\mathrm{C} 3$ & $1.328(2)$ & $\mathrm{C} 13-\mathrm{H} 13 \mathrm{~B}$ & 0.9700 \\
$\mathrm{~N} 2-\mathrm{C} 4$ & $1.352(2)$ & $\mathrm{C} 14-\mathrm{C} 15$ & $1.394(3)$ \\
$\mathrm{N} 3-\mathrm{C} 7$ & $1.374(2)$ & $\mathrm{C} 14-\mathrm{C} 19$ & $1.398(3)$ \\
$\mathrm{N} 3-\mathrm{C} 6$ & $1.453(2)$ & $\mathrm{C} 15-\mathrm{C} 16$ & $1.376(3)$ \\
$\mathrm{N} 3-\mathrm{C} 5$ & $1.463(2)$ & $\mathrm{C} 15-\mathrm{H} 15$ & 0.9300 \\
$\mathrm{~N} 4-\mathrm{C} 22$ & $1.415(3)$ & $\mathrm{C} 16-\mathrm{C} 17$ & $1.367(4)$ \\
$\mathrm{N} 4-\mathrm{C} 28$ & $1.449(3)$ & $\mathrm{C} 16-\mathrm{H} 16$ & 0.9300 \\
$\mathrm{~N} 4-\mathrm{C} 21$ & $1.453(3)$ & $\mathrm{C} 17-\mathrm{C} 18$ & $1.376(4)$ \\
$\mathrm{N} 5-\mathrm{C} 14$ & $1.376(3)$ & $\mathrm{C} 17-\mathrm{H} 17$ & 0.9300 \\
$\mathrm{~N} 5-\mathrm{C} 13$ & $1.449(3)$ & $\mathrm{C} 18-\mathrm{C} 19$ & $1.367(4)$ \\
$\mathrm{N} 5-\mathrm{C} 20$ & $1.454(3)$ & $\mathrm{C} 18-\mathrm{H} 18$ & 0.9300 \\
$\mathrm{C} 1-\mathrm{C} 4$ & $1.397(3)$ & $\mathrm{C} 19-\mathrm{H} 19$ & 0.9300 \\
$\mathrm{C} 1-\mathrm{C} 13$ & $1.527(3)$ & $\mathrm{C} 20-\mathrm{H} 20 \mathrm{~A}$ & 0.9600 \\
$\mathrm{C} 2-\mathrm{C} 3$ & $1.377(2)$ & $\mathrm{C} 20-\mathrm{H} 20 \mathrm{~B}$ & 0.9600 \\
$\mathrm{C} 2-\mathrm{C} 5$ & $1.483(3)$ & $\mathrm{C} 20-\mathrm{H} 20 \mathrm{C}$ & 0.9600 \\
$\mathrm{C} 3-\mathrm{C} 6$ & $1.498(3)$ & $\mathrm{C} 21-\mathrm{H} 21 \mathrm{~A}$ & 0.9700 \\
$\mathrm{C} 4-\mathrm{C} 21$ & $1.515(3)$ & $\mathrm{C} 21-\mathrm{H} 21 \mathrm{~B}$ & 0.9700 \\
$\mathrm{C} 5-\mathrm{H} 5 \mathrm{~A}$ & 0.9700 & $\mathrm{C} 22-\mathrm{C} 23$ & $1.378(3)$ \\
$\mathrm{C} 5-\mathrm{H} 5 \mathrm{~B}$ & 0.9700 & $\mathrm{C} 22-\mathrm{C} 27$ & $1.395(3)$
\end{tabular}




\begin{tabular}{|c|c|c|c|}
\hline C6-H6A & 0.9700 & $\mathrm{C} 23-\mathrm{C} 24$ & $1.390(3)$ \\
\hline $\mathrm{C} 6-\mathrm{H} 6 \mathrm{~B}$ & 0.9700 & $\mathrm{C} 23-\mathrm{H} 23$ & 0.9300 \\
\hline $\mathrm{C} 7-\mathrm{C} 8$ & $1.392(3)$ & $\mathrm{C} 24-\mathrm{C} 25$ & $1.373(4)$ \\
\hline $\mathrm{C} 7-\mathrm{C} 12$ & $1.401(3)$ & $\mathrm{C} 24-\mathrm{H} 24$ & 0.9300 \\
\hline $\mathrm{C} 8-\mathrm{C} 9$ & $1.385(3)$ & $\mathrm{C} 25-\mathrm{C} 26$ & $1.366(4)$ \\
\hline $\mathrm{C} 8-\mathrm{H} 8$ & 0.9300 & $\mathrm{C} 25-\mathrm{H} 25$ & 0.9300 \\
\hline $\mathrm{C} 9-\mathrm{C} 10$ & $1.370(3)$ & $\mathrm{C} 26-\mathrm{C} 27$ & $1.378(3)$ \\
\hline C9-H9 & 0.9300 & $\mathrm{C} 26-\mathrm{H} 26$ & 0.9300 \\
\hline $\mathrm{C} 10-\mathrm{C} 11$ & $1.373(3)$ & $\mathrm{C} 27-\mathrm{H} 27$ & 0.9300 \\
\hline $\mathrm{C} 10-\mathrm{H} 10$ & 0.9300 & $\mathrm{C} 28-\mathrm{H} 28 \mathrm{~A}$ & 0.9600 \\
\hline $\mathrm{C} 11-\mathrm{C} 12$ & $1.380(3)$ & $\mathrm{C} 28-\mathrm{H} 28 \mathrm{~B}$ & 0.9600 \\
\hline $\mathrm{C} 11-\mathrm{H} 11$ & 0.9300 & $\mathrm{C} 28-\mathrm{H} 28 \mathrm{C}$ & 0.9600 \\
\hline $\mathrm{C} 2-\mathrm{N} 1-\mathrm{C} 1$ & $114.96(16)$ & $\mathrm{C} 1-\mathrm{C} 13-\mathrm{H} 13 \mathrm{~B}$ & 108.9 \\
\hline $\mathrm{C} 3-\mathrm{N} 2-\mathrm{C} 4$ & $115.23(17)$ & $\mathrm{H} 13 \mathrm{~A}-\mathrm{C} 13-\mathrm{H} 13 \mathrm{~B}$ & 107.7 \\
\hline $\mathrm{C} 7-\mathrm{N} 3-\mathrm{C} 6$ & $122.57(16)$ & $\mathrm{N} 5-\mathrm{C} 14-\mathrm{C} 15$ & $121.3(2)$ \\
\hline $\mathrm{C} 7-\mathrm{N} 3-\mathrm{C} 5$ & $123.11(17)$ & $\mathrm{N} 5-\mathrm{C} 14-\mathrm{C} 19$ & $121.3(2)$ \\
\hline $\mathrm{C} 6-\mathrm{N} 3-\mathrm{C} 5$ & $113.64(16)$ & $\mathrm{C} 15-\mathrm{C} 14-\mathrm{C} 19$ & $117.3(3)$ \\
\hline $\mathrm{C} 22-\mathrm{N} 4-\mathrm{C} 28$ & $118.35(19)$ & $\mathrm{C} 16-\mathrm{C} 15-\mathrm{C} 14$ & $120.9(3)$ \\
\hline $\mathrm{C} 22-\mathrm{N} 4-\mathrm{C} 21$ & $117.9(2)$ & $\mathrm{C} 16-\mathrm{C} 15-\mathrm{H} 15$ & 119.5 \\
\hline $\mathrm{C} 28-\mathrm{N} 4-\mathrm{C} 21$ & $114.48(18)$ & $\mathrm{C} 14-\mathrm{C} 15-\mathrm{H} 15$ & 119.5 \\
\hline $\mathrm{C} 14-\mathrm{N} 5-\mathrm{C} 13$ & $120.75(19)$ & $\mathrm{C} 17-\mathrm{C} 16-\mathrm{C} 15$ & $121.1(3)$ \\
\hline $\mathrm{C} 14-\mathrm{N} 5-\mathrm{C} 20$ & $119.8(2)$ & $\mathrm{C} 17-\mathrm{C} 16-\mathrm{H} 16$ & 119.4 \\
\hline $\mathrm{C} 13-\mathrm{N} 5-\mathrm{C} 20$ & $117.9(2)$ & $\mathrm{C} 15-\mathrm{C} 16-\mathrm{H} 16$ & 119.4 \\
\hline $\mathrm{N} 1-\mathrm{C} 1-\mathrm{C} 4$ & $122.07(18)$ & $\mathrm{C} 16-\mathrm{C} 17-\mathrm{C} 18$ & $118.5(3)$ \\
\hline $\mathrm{N} 1-\mathrm{C} 1-\mathrm{C} 13$ & $115.34(18)$ & $\mathrm{C} 16-\mathrm{C} 17-\mathrm{H} 17$ & 120.8 \\
\hline $\mathrm{C} 4-\mathrm{C} 1-\mathrm{C} 13$ & $122.5(2)$ & $\mathrm{C} 18-\mathrm{C} 17-\mathrm{H} 17$ & 120.8 \\
\hline $\mathrm{N} 1-\mathrm{C} 2-\mathrm{C} 3$ & $122.80(19)$ & $\mathrm{C} 19-\mathrm{C} 18-\mathrm{C} 17$ & $121.6(3)$ \\
\hline $\mathrm{N} 1-\mathrm{C} 2-\mathrm{C} 5$ & $125.96(18)$ & $\mathrm{C} 19-\mathrm{C} 18-\mathrm{H} 18$ & 119.2 \\
\hline $\mathrm{C} 3-\mathrm{C} 2-\mathrm{C} 5$ & $111.24(18)$ & $\mathrm{C} 17-\mathrm{C} 18-\mathrm{H} 18$ & 119.2 \\
\hline $\mathrm{N} 2-\mathrm{C} 3-\mathrm{C} 2$ & $123.17(19)$ & $\mathrm{C} 18-\mathrm{C} 19-\mathrm{C} 14$ & $120.6(3)$ \\
\hline $\mathrm{N} 2-\mathrm{C} 3-\mathrm{C} 6$ & $126.31(18)$ & $\mathrm{C} 18-\mathrm{C} 19-\mathrm{H} 19$ & 119.7 \\
\hline $\mathrm{C} 2-\mathrm{C} 3-\mathrm{C} 6$ & $110.52(19)$ & $\mathrm{C} 14-\mathrm{C} 19-\mathrm{H} 19$ & 119.7 \\
\hline $\mathrm{N} 2-\mathrm{C} 4-\mathrm{C} 1$ & $121.65(19)$ & $\mathrm{N} 5-\mathrm{C} 20-\mathrm{H} 20 \mathrm{~A}$ & 109.5 \\
\hline $\mathrm{N} 2-\mathrm{C} 4-\mathrm{C} 21$ & $115.81(19)$ & $\mathrm{N} 5-\mathrm{C} 20-\mathrm{H} 20 \mathrm{~B}$ & 109.5 \\
\hline $\mathrm{C} 1-\mathrm{C} 4-\mathrm{C} 21$ & $122.52(19)$ & $\mathrm{H} 20 \mathrm{~A}-\mathrm{C} 20-\mathrm{H} 20 \mathrm{~B}$ & 109.5 \\
\hline $\mathrm{N} 3-\mathrm{C} 5-\mathrm{C} 2$ & $102.26(16)$ & $\mathrm{N} 5-\mathrm{C} 20-\mathrm{H} 20 \mathrm{C}$ & 109.5 \\
\hline $\mathrm{N} 3-\mathrm{C} 5-\mathrm{H} 5 \mathrm{~A}$ & 111.3 & $\mathrm{H} 20 \mathrm{~A}-\mathrm{C} 20-\mathrm{H} 20 \mathrm{C}$ & 109.5 \\
\hline $\mathrm{C} 2-\mathrm{C} 5-\mathrm{H} 5 \mathrm{~A}$ & 111.3 & $\mathrm{H} 20 \mathrm{~B}-\mathrm{C} 20-\mathrm{H} 20 \mathrm{C}$ & 109.5 \\
\hline $\mathrm{N} 3-\mathrm{C} 5-\mathrm{H} 5 \mathrm{~B}$ & 111.3 & $\mathrm{~N} 4-\mathrm{C} 21-\mathrm{C} 4$ & $112.0(2)$ \\
\hline $\mathrm{C} 2-\mathrm{C} 5-\mathrm{H} 5 \mathrm{~B}$ & 111.3 & $\mathrm{~N} 4-\mathrm{C} 21-\mathrm{H} 21 \mathrm{~A}$ & 109.2 \\
\hline $\mathrm{H} 5 \mathrm{~A}-\mathrm{C} 5-\mathrm{H} 5 \mathrm{~B}$ & 109.2 & $\mathrm{C} 4-\mathrm{C} 21-\mathrm{H} 21 \mathrm{~A}$ & 109.2 \\
\hline $\mathrm{N} 3-\mathrm{C} 6-\mathrm{C} 3$ & $102.22(16)$ & $\mathrm{N} 4-\mathrm{C} 21-\mathrm{H} 21 \mathrm{~B}$ & 109.2 \\
\hline $\mathrm{N} 3-\mathrm{C} 6-\mathrm{H} 6 \mathrm{~A}$ & 111.3 & $\mathrm{C} 4-\mathrm{C} 21-\mathrm{H} 21 \mathrm{~B}$ & 109.2 \\
\hline $\mathrm{C} 3-\mathrm{C} 6-\mathrm{H} 6 \mathrm{~A}$ & 111.3 & $\mathrm{H} 21 \mathrm{~A}-\mathrm{C} 21-\mathrm{H} 21 \mathrm{~B}$ & 107.9 \\
\hline N3- $66-\mathrm{H} 6 \mathrm{~B}$ & 111.3 & $\mathrm{C} 23-\mathrm{C} 22-\mathrm{C} 27$ & $117.8(2)$ \\
\hline $\mathrm{C} 3-\mathrm{C} 6-\mathrm{H} 6 \mathrm{~B}$ & 111.3 & $\mathrm{C} 23-\mathrm{C} 22-\mathrm{N} 4$ & $123.5(2)$ \\
\hline $\mathrm{H} 6 \mathrm{~A}-\mathrm{C} 6-\mathrm{H} 6 \mathrm{~B}$ & 109.2 & $\mathrm{C} 27-\mathrm{C} 22-\mathrm{N} 4$ & $118.7(3)$ \\
\hline
\end{tabular}




\begin{tabular}{|c|c|}
\hline $\mathrm{N} 3-\mathrm{C} 7-\mathrm{C} 8$ & $121.19(19)$ \\
\hline $\mathrm{N} 3-\mathrm{C} 7-\mathrm{C} 12$ & $120.9(2)$ \\
\hline $\mathrm{C} 8-\mathrm{C} 7-\mathrm{C} 12$ & $117.9(2)$ \\
\hline $\mathrm{C} 9-\mathrm{C} 8-\mathrm{C} 7$ & $120.4(2)$ \\
\hline $\mathrm{C} 9-\mathrm{C} 8-\mathrm{H} 8$ & 119.8 \\
\hline $\mathrm{C} 7-\mathrm{C} 8-\mathrm{H} 8$ & 119.8 \\
\hline $\mathrm{C} 10-\mathrm{C} 9-\mathrm{C} 8$ & $121.2(2)$ \\
\hline $\mathrm{C} 10-\mathrm{C} 9-\mathrm{H} 9$ & 119.4 \\
\hline $\mathrm{C} 8-\mathrm{C} 9-\mathrm{H} 9$ & 119.4 \\
\hline $\mathrm{C} 9-\mathrm{C} 10-\mathrm{C} 11$ & $119.1(2)$ \\
\hline $\mathrm{C} 9-\mathrm{C} 10-\mathrm{H} 10$ & 120.4 \\
\hline $\mathrm{C} 11-\mathrm{C} 10-\mathrm{H} 10$ & 120.4 \\
\hline $\mathrm{C} 10-\mathrm{C} 11-\mathrm{C} 12$ & $120.8(2)$ \\
\hline $\mathrm{C} 10-\mathrm{C} 11-\mathrm{H} 11$ & 119.6 \\
\hline $\mathrm{C} 12-\mathrm{C} 11-\mathrm{H} 11$ & 119.6 \\
\hline $\mathrm{C} 11-\mathrm{C} 12-\mathrm{C} 7$ & $120.6(2)$ \\
\hline $\mathrm{C} 11-\mathrm{C} 12-\mathrm{H} 12$ & 119.7 \\
\hline $\mathrm{C} 7-\mathrm{C} 12-\mathrm{H} 12$ & 119.7 \\
\hline $\mathrm{N} 5-\mathrm{C} 13-\mathrm{C} 1$ & $113.53(19)$ \\
\hline $\mathrm{N} 5-\mathrm{C} 13-\mathrm{H} 13 \mathrm{~A}$ & 108.9 \\
\hline $\mathrm{C} 1-\mathrm{C} 13-\mathrm{H} 13 \mathrm{~A}$ & 108.9 \\
\hline $\mathrm{N} 5-\mathrm{C} 13-\mathrm{H} 13 \mathrm{~B}$ & 108.9 \\
\hline $\mathrm{C} 2-\mathrm{N} 1-\mathrm{C} 1-\mathrm{C} 4$ & $-2.9(3)$ \\
\hline $\mathrm{C} 2-\mathrm{N} 1-\mathrm{C} 1-\mathrm{C} 13$ & $-180.0(2)$ \\
\hline $\mathrm{C} 1-\mathrm{N} 1-\mathrm{C} 2-\mathrm{C} 3$ & $0.0(3)$ \\
\hline $\mathrm{C} 1-\mathrm{N} 1-\mathrm{C} 2-\mathrm{C} 5$ & $-179.5(2)$ \\
\hline $\mathrm{C} 4-\mathrm{N} 2-\mathrm{C} 3-\mathrm{C} 2$ & $-2.3(3)$ \\
\hline $\mathrm{C} 4-\mathrm{N} 2-\mathrm{C} 3-\mathrm{C} 6$ & $178.1(2)$ \\
\hline $\mathrm{N} 1-\mathrm{C} 2-\mathrm{C} 3-\mathrm{N} 2$ & $2.8(3)$ \\
\hline $\mathrm{C} 5-\mathrm{C} 2-\mathrm{C} 3-\mathrm{N} 2$ & $-177.7(2)$ \\
\hline $\mathrm{N} 1-\mathrm{C} 2-\mathrm{C} 3-\mathrm{C} 6$ & $-177.6(2)$ \\
\hline $\mathrm{C} 5-\mathrm{C} 2-\mathrm{C} 3-\mathrm{C} 6$ & $2.0(3)$ \\
\hline $\mathrm{C} 3-\mathrm{N} 2-\mathrm{C} 4-\mathrm{C} 1$ & $-0.6(3)$ \\
\hline $\mathrm{C} 3-\mathrm{N} 2-\mathrm{C} 4-\mathrm{C} 21$ & $177.8(2)$ \\
\hline $\mathrm{N} 1-\mathrm{C} 1-\mathrm{C} 4-\mathrm{N} 2$ & $3.4(3)$ \\
\hline $\mathrm{C} 13-\mathrm{C} 1-\mathrm{C} 4-\mathrm{N} 2$ & $-179.8(2)$ \\
\hline $\mathrm{N} 1-\mathrm{C} 1-\mathrm{C} 4-\mathrm{C} 21$ & $-174.9(2)$ \\
\hline $\mathrm{C} 13-\mathrm{C} 1-\mathrm{C} 4-\mathrm{C} 21$ & $2.0(3)$ \\
\hline $\mathrm{C} 7-\mathrm{N} 3-\mathrm{C} 5-\mathrm{C} 2$ & $-173.1(2)$ \\
\hline $\mathrm{C} 6-\mathrm{N} 3-\mathrm{C} 5-\mathrm{C} 2$ & $-2.3(2)$ \\
\hline $\mathrm{N} 1-\mathrm{C} 2-\mathrm{C} 5-\mathrm{N} 3$ & $179.7(2)$ \\
\hline $\mathrm{C} 3-\mathrm{C} 2-\mathrm{C} 5-\mathrm{N} 3$ & $0.1(2)$ \\
\hline $\mathrm{C} 7-\mathrm{N} 3-\mathrm{C} 6-\mathrm{C} 3$ & $174.2(2)$ \\
\hline $\mathrm{C} 5-\mathrm{N} 3-\mathrm{C} 6-\mathrm{C} 3$ & $3.3(2)$ \\
\hline $\mathrm{N} 2-\mathrm{C} 3-\mathrm{C} 6-\mathrm{N} 3$ & $176.5(2)$ \\
\hline $\mathrm{C} 2-\mathrm{C} 3-\mathrm{C} 6-\mathrm{N} 3$ & $-3.2(2)$ \\
\hline $\mathrm{C} 6-\mathrm{N} 3-\mathrm{C} 7-\mathrm{C} 8$ & $-175.5(2)$ \\
\hline
\end{tabular}

$\mathrm{C} 22-\mathrm{C} 23-\mathrm{C} 24 \quad 120.7$ (3)

$\mathrm{C} 22-\mathrm{C} 23-\mathrm{H} 23 \quad 119.6$

$\mathrm{C} 24-\mathrm{C} 23-\mathrm{H} 23 \quad 119.6$

$\mathrm{C} 25-\mathrm{C} 24-\mathrm{C} 23 \quad 121.0$ (3)

$\mathrm{C} 25-\mathrm{C} 24-\mathrm{H} 24 \quad 119.5$

$\mathrm{C} 23-\mathrm{C} 24-\mathrm{H} 24$

$\mathrm{C} 26-\mathrm{C} 25-\mathrm{C} 24$

$\mathrm{C} 26-\mathrm{C} 25-\mathrm{H} 25 \quad 120.8$

$\mathrm{C} 24-\mathrm{C} 25-\mathrm{H} 25 \quad 120.8$

$\mathrm{C} 25-\mathrm{C} 26-\mathrm{C} 27 \quad 121.5$ (3)

$\mathrm{C} 25-\mathrm{C} 26-\mathrm{H} 26 \quad 119.3$

$\mathrm{C} 27-\mathrm{C} 26-\mathrm{H} 26 \quad 119.3$

$\mathrm{C} 26-\mathrm{C} 27-\mathrm{C} 22$

$\mathrm{C} 26-\mathrm{C} 27-\mathrm{H} 27 \quad 119.7$

$\mathrm{C} 22-\mathrm{C} 27-\mathrm{H} 27 \quad 119.7$

$\mathrm{N} 4-\mathrm{C} 28-\mathrm{H} 28 \mathrm{~A} \quad 109.5$

$\mathrm{N} 4-\mathrm{C} 28-\mathrm{H} 28 \mathrm{~B} \quad 109.5$

$\mathrm{H} 28 \mathrm{~A}-\mathrm{C} 28-\mathrm{H} 28 \mathrm{~B} \quad 109.5$

$\mathrm{N} 4-\mathrm{C} 28-\mathrm{H} 28 \mathrm{C} \quad 109.5$

$\mathrm{H} 28 \mathrm{~A}-\mathrm{C} 28-\mathrm{H} 28 \mathrm{C} \quad 109.5$

$\mathrm{H} 28 \mathrm{~B}-\mathrm{C} 28-\mathrm{H} 28 \mathrm{C} \quad 109.5$

$\begin{array}{ll}\mathrm{N} 3-\mathrm{C} 7-\mathrm{C} 12-\mathrm{C} 11 & 178.6(2) \\ \mathrm{C} 8-\mathrm{C} 7-\mathrm{C} 12-\mathrm{C} 11 & -0.4(3) \\ \mathrm{C} 14-\mathrm{N} 5-\mathrm{C} 13-\mathrm{C} 1 & -75.0(2) \\ \mathrm{C} 20-\mathrm{N} 5-\mathrm{C} 13-\mathrm{C} 1 & 91.0(3) \\ \mathrm{N} 1-\mathrm{C} 1-\mathrm{C} 13-\mathrm{N} 5 & -37.6(3) \\ \mathrm{C} 4-\mathrm{C} 1-\mathrm{C} 13-\mathrm{N} 5 & 145.3(2) \\ \mathrm{C} 13-\mathrm{N} 5-\mathrm{C} 14-\mathrm{C} 15 & -7.6(3) \\ \mathrm{C} 20-\mathrm{N} 5-\mathrm{C} 14-\mathrm{C} 15 & -173.29(19) \\ \mathrm{C} 13-\mathrm{N} 5-\mathrm{C} 14-\mathrm{C} 19 & 173.80(19) \\ \mathrm{C} 20-\mathrm{N} 5-\mathrm{C} 14-\mathrm{C} 19 & 8.1(3) \\ \mathrm{N} 5-\mathrm{C} 14-\mathrm{C} 15-\mathrm{C} 16 & -178.5(2) \\ \mathrm{C} 19-\mathrm{C} 14-\mathrm{C} 15-\mathrm{C} 16 & 0.2(3) \\ \mathrm{C} 14-\mathrm{C} 15-\mathrm{C} 16-\mathrm{C} 17 & -0.6(4) \\ \mathrm{C} 15-\mathrm{C} 16-\mathrm{C} 17-\mathrm{C} 18 & 0.9(4) \\ \mathrm{C} 16-\mathrm{C} 17-\mathrm{C} 18-\mathrm{C} 19 & -1.0(4) \\ \mathrm{C} 17-\mathrm{C} 18-\mathrm{C} 19-\mathrm{C} 14 & 0.7(4) \\ \mathrm{N} 5-\mathrm{C} 14-\mathrm{C} 19-\mathrm{C} 18 & 178.4(2) \\ \mathrm{C} 15-\mathrm{C} 14-\mathrm{C} 19-\mathrm{C} 18 & -0.3(3) \\ \mathrm{C} 22-\mathrm{N} 4-\mathrm{C} 21-\mathrm{C} 4 & 154.45(18) \\ \mathrm{C} 28-\mathrm{N} 4-\mathrm{C} 21-\mathrm{C} 4 & -59.3(2) \\ \mathrm{N} 2-\mathrm{C} 4-\mathrm{C} 21-\mathrm{N} 4 & 103.8(2) \\ \mathrm{C} 1-\mathrm{C} 4-\mathrm{C} 21-\mathrm{N} 4 & -77.8(3) \\ \mathrm{C} 28-\mathrm{N} 4-\mathrm{C} 22-\mathrm{C} 23 & -146.2(2) \\ \mathrm{C} 21-\mathrm{N} 4-\mathrm{C} 22-\mathrm{C} 23 & -1.2(3) \\ \mathrm{C} 28-\mathrm{N} 4-\mathrm{C} 22-\mathrm{C} 27 & 36.3(3) \\ \end{array}$




$\begin{array}{llll}\mathrm{C} 5-\mathrm{N} 3-\mathrm{C} 7-\mathrm{C} 8 & -5.5(3) & \mathrm{C} 21-\mathrm{N} 4-\mathrm{C} 22-\mathrm{C} 27 & -178.72(19) \\ \mathrm{C} 6-\mathrm{N} 3-\mathrm{C} 7-\mathrm{C} 12 & 5.6(3) & \mathrm{C} 27-\mathrm{C} 22-\mathrm{C} 23-\mathrm{C} 24 & 1.6(3) \\ \mathrm{C} 5-\mathrm{N} 3-\mathrm{C} 7-\mathrm{C} 12 & 175.6(2) & \mathrm{N} 4-\mathrm{C} 22-\mathrm{C} 23-\mathrm{C} 24 & -175.92(19) \\ \mathrm{N} 3-\mathrm{C} 7-\mathrm{C} 8-\mathrm{C} 9 & -178.8(2) & \mathrm{C} 22-\mathrm{C} 23-\mathrm{C} 24-\mathrm{C} 25 & -0.6(4) \\ \mathrm{C} 12-\mathrm{C} 7-\mathrm{C} 8-\mathrm{C} 9 & 0.1(3) & \mathrm{C} 23-\mathrm{C} 24-\mathrm{C} 25-\mathrm{C} 26 & -0.8(4) \\ \mathrm{C} 7-\mathrm{C} 8-\mathrm{C} 9-\mathrm{C} 10 & 0.2(4) & \mathrm{C} 24-\mathrm{C} 25-\mathrm{C} 26-\mathrm{C} 27 & 1.3(4) \\ \mathrm{C} 8-\mathrm{C} 9-\mathrm{C} 10-\mathrm{C} 11 & -0.3(4) & \mathrm{C} 25-\mathrm{C} 26-\mathrm{C} 27-\mathrm{C} 22 & -0.3(4) \\ \mathrm{C} 9-\mathrm{C} 10-\mathrm{C} 11-\mathrm{C} 12 & 0.1(4) & \mathrm{C} 23-\mathrm{C} 22-\mathrm{C} 27-\mathrm{C} 26 & -1.2(3) \\ \mathrm{C} 10-\mathrm{C} 11-\mathrm{C} 12-\mathrm{C} 7 & 0.3(4) & \mathrm{N} 4-\mathrm{C} 22-\mathrm{C} 27-\mathrm{C} 26 & 176.5(2)\end{array}$

Hydrogen-bond geometry $\left(A,{ }^{\circ}\right)$

$C g 2$ and $C g 3$ are the centroids of rings N1/N2/C1-C4 and C7-C12, respectively.

\begin{tabular}{lllll}
\hline$D-\mathrm{H} \cdots A$ & $D-\mathrm{H}$ & $\mathrm{H} \cdots A$ & $D \cdots A$ & $D-\mathrm{H} \cdots A$ \\
\hline $\mathrm{C} 15-\mathrm{H} 15 \cdots \mathrm{N} 4$ & 0.93 & 2.61 & $3.542(3)$ & 175 \\
$\mathrm{C} 28-\mathrm{H} 28 B \cdots \mathrm{N} 2$ & 0.96 & 2.59 & $3.323(3)$ & 133 \\
$\mathrm{C} 6-\mathrm{H} 6 B \cdots C g 2^{\mathrm{i}}$ & 0.97 & 2.82 & $3.601(2)$ & 138 \\
$\mathrm{C} 23-\mathrm{H} 23 \cdots C g 3^{\mathrm{i}}$ & 0.93 & 2.97 & $3.881(3)$ & 168
\end{tabular}

Symmetry code: (i) $-x+1,-y+2,-z$. 